\title{
A review on chemical and mechanical phenomena at the wafer interface during chemical mechanical planarization
}

\author{
Jihoon $\mathrm{SeO}^{\mathrm{a})}(\mathrm{it}$ \\ Department of Chemical and Biomolecular Engineering and Center for Advanced Materials Processing, Clarkson University, Potsdam 13699, \\ New York, USA \\ a)Jihoon Seo jseo@clarkson.edu
}

Received: 5 June 2020; accepted: 21 July 2020; published online: 1 February 2021

As the minimum feature size of integrated circuit elements has shrunk below $7 \mathrm{~nm}$, chemical mechanical planarization (CMP) technology has grown by leaps and bounds over the past several decades. There has been a growing interest in understanding the fundamental science and technology of CMP, which has continued to lag behind advances in technology. This review paper provides a comprehensive overview of various chemical and mechanical phenomena such as contact mechanics, lubrication models, chemical reaction that occur between slurry components and films being polished, electrochemical reactions, adsorption behavior and mechanism, temperature effects, and the complex interactions occurring at the wafer interface during polishing. It also provides important insights into new strategies and novel concepts for next-generation CMP slurries. Finally, the challenges and future research directions related to the chemical and mechanical process and slurry chemistry are highlighted.

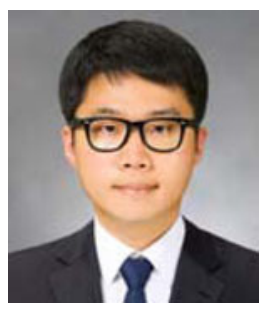

\begin{abstract}
Dr. Jihoon Seo is a research assistant professor of Chemical and Biomolecular Engineering at Clarkson University, USA. He received his Bachelor of Science degree in materials science and engineering from Hanyang University (HYU), Seoul, Korea in 2010, and his Ph.D. degree in energy engineering from HYU in 2017 under the supervision of Prof. Ungyu Paik. He was a post-doctoral research associate at the Center for Advanced Materials Processing at Clarkson University from October 2017 to February 2018, where he was mentored by Prof. S.V. Babu. Dr. Seo has worked on many research projects in various fields ranging from fundamental principles (e.g., surface chemistry, colloidal chemistry, nanotechnology, and electrochemistry) to practical applications [e.g., Chemical Mechanical Planarization (CMP) and post-CMP cleaning]. In particular, throughout his career, his research interests have focused on the development of CMP slurries and post-CMP cleaning solutions through a fundamental understanding of colloid and surface chemistry, liquid-solid interface chemistry, and the electrochemistry of various metallic and dielectric thin films and heterogeneous structures containing them. The main objective of his research is not only to understand the various phenomena occurring at the wafer interface during polishing and cleaning but also to provide researchers who study CMP and post-CMP cleaning processes with new strategies and novel concepts for the development of advanced processes for future devices.
\end{abstract}

\section{Introduction}

Chemical mechanical planarization (CMP) is one of the most critical processes to achieve multilevel metallization and incorporation of gate and channel materials during integrated circuit (IC) fabrication [1, 2]. Applications of CMP can be found in three main areas of IC manufacturing that include forming the transistors (front-end-of-line, FEOL), the local electrical connections between transistors (middle-of-line, MOL), and the interconnect structures (back-end-of-line, BEOL) [1, 2]. The FEOL process contains all the necessary steps to build the device architecture with a variety of CMP steps for different layer combinations of $\mathrm{SiO}_{2}, \mathrm{Si}_{3} \mathrm{~N}_{4}$, and poly-Si stop layers, $\mathrm{SiC}$, $\mathrm{SiCN}$, etc. and the high-k/metal gate structures $[3,4]$. The MOL processes are introduced to connect the individual 
transistors. W and Co have attracted significant attention as an electrically conducting material for local interconnects for the MOL process steps [5]. In the BEOL process steps, all the number of devices are interconnected by sequentially constructing multilevel $\mathrm{Cu}$ wires and insulating layers [6]. CMP has been an enabling technology in the FEOL, the MOL, and the BEOL processes by achieving the desired removal rates, selectivity, and ultimately planarity with different substrate materials. Each CMP process requires different removal rates, selectivities, and process conditions. As feature sizes continue to decrease, requiring the fabrication of more complex geometries, tunable and selective polishing of different films has become even more critical. New CMP steps, including new materials and complex structures, are proposed and present even more stringent requirements.

The CMP process has become increasingly sophisticated over the years. Many of the underlying fundamental mechanisms are still not well understood. Indeed, CMP technology is advancing faster than our understanding of it. The evolution of high-volume-production CMP processes has its marketdriven schedules that are ahead of our understanding of the fundamental principles/technologies. Thus, the demand for understanding of various phenomena that occur during CMP is increasing in both industrial and academic research.

CMP utilizes a synergistic interplay of chemical and mechanical interactions to achieve the desired removal rates, selectivity, and ultimately planarity with different substrate materials. During this process, the substrate is pressed down typically onto a grooved polymeric, most commonly polyurethane pad with controlled hardness and texture under an applied force (Fig. 1). The polishing slurry is transported into the grooves, pores, and texture of the polyurethane pad by rotating the pad at high speed, which generates the chemical and mechanical actions involving three-body (slurry/polishing pad/wafer) interactions (Fig. 1). Many factors including CMP consumables and their process/tool conditions can impact the polishing performance. In this review, I have focused mainly on CMP slurries and their relation to chemical and mechanical aspects of the CMP process. In general, the slurries for the dielectric CMP process are composed of abrasives, dispersant, passivation agent for high selectivity, $\mathrm{pH}$ adjuster, and deionized water. In contrast with dielectric materials, metal CMP is governed by the electrochemical behavior of metal films in the presence of oxidizer, complexing agents, and corrosion inhibitors. Metal CMP slurries may contain additional chemical reagents like oxidizer, complexing agent, and corrosion inhibitor. Typical slurry components for dielectric and metal CMP processes are summarized in Table 1. Slurry formulations not only are different depending on the materials to be polished but also need to be optimized to meet the stringent process requirements $[7,8]$. Their characteristics dominate the various interactions that occur at the slurry/pad-wafer interface.

This review presents and discusses the most significant advances with respect to chemical and mechanical phenomena at the wafer interface during CMP. Chemical and mechanical phenomena such as contact mechanics, lubrication models, the chemical reactions between slurry components and films

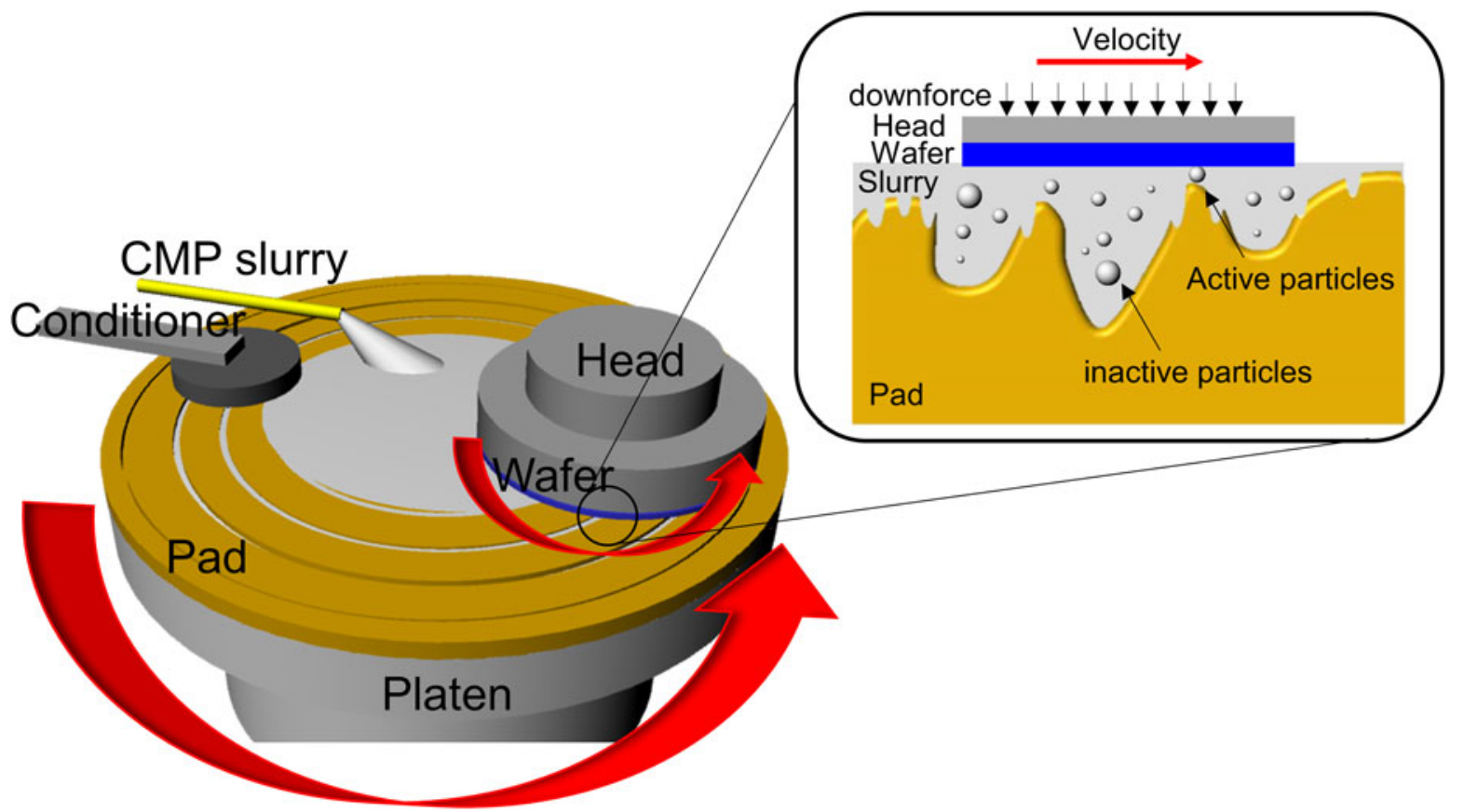

Figure 1: Schematic diagram of a typical CMP tool and three-body interactions occurring in the wafer/abrasive/pad contact region. 
TABLE 1. Typical CMP slurry components.

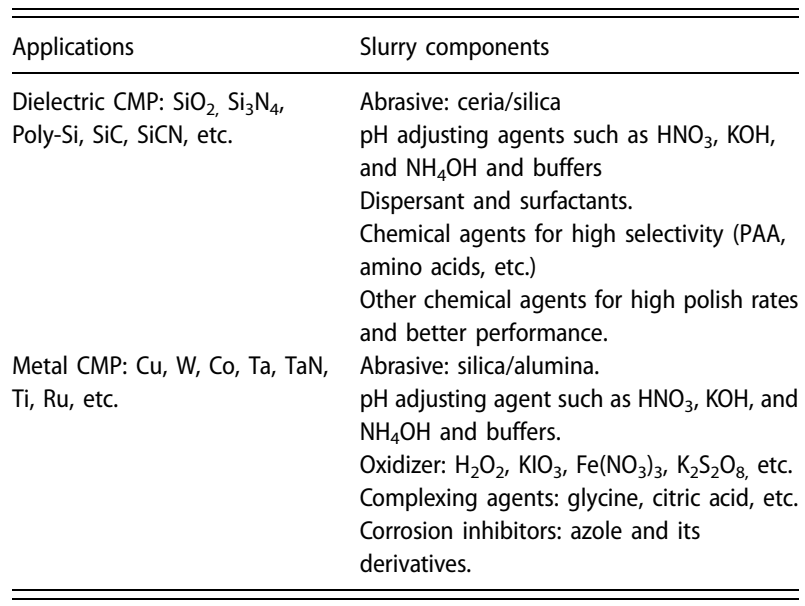

being polished, electrochemical reactions, adsorption behavior and mechanisms, temperature effects, and their complex interactions will be discussed. Also, the final section provides an overview of recently developed real-time in situ techniques to study some of the phenomena that occur at the slurry/padwafer interface during polishing.

\section{Models Based on Contact Mechanics}

Material removal occurs when there is direct contact between an abrasive particle and wafer surface during polishing. Models based on contact mechanics were proposed to explain the mechanical aspects of the material removal mechanism. Assuming that all particles are involved in material removal, their contact area on the particle-wafer surface is given as $A \propto C^{1 / 3} \cdot d^{-1 / 3}$ where $C$ is the concentration of the abrasive particles and $d$ is the abrasive diameter [9]. However, only abrasive particles larger than the gap between the polishing pad and the wafer surface, termed as "active" particles, can contribute to material removal (Fig. 1) [10]. Of course, a larger gap will result in less interaction between particles and wafer. Luo and Dornfeld assumed that only the plastic, but not elastic, deformation caused by the indentation of the active particles into the wafer surface results in material removal. They developed a mathematical model to predict the material removal rates considering the characteristics of the active abrasive particles (size, size distribution, and concentration), pad hardness, and CMP process conditions $[10,11]$. The real contact area $\left(A_{\mathrm{r}}\right)$ at the wafer-particle interface considering pad asperities with a Greenwood-Williamson approach was proposed by Lee et al. [12], which can be expressed as $A_{\mathrm{r}}=\left(f_{\mathrm{s}} / C\right)$ $\left(R_{\mathrm{p}} / \sigma_{\mathrm{p}}\right)^{1 / 2}\left(P A_{\mathrm{w}} / E_{\mathrm{pw}}\right)$ where $R_{\mathrm{p}}$ is the average radius of curvature of pad asperity, $\sigma_{\mathrm{p}}$ is the standard deviation of the pad asperity height, $P$ is the applied pressure, $A_{\mathrm{w}}$ is the nominal area of the wafer surface, $E_{\mathrm{pw}}$ is the compose Young's modulus of the pad and the wafer, $f_{\mathrm{s}}$ is the area density of up area of the grooved pad, and $C$ is a constant in the range of 0.3-0.4. Lee et al. reported that the real contact area ratio (real contact area/nominal contact area) continued to decrease over the CMP process, leading to the deterioration in the material removal rates [13]. Park and Jeong suggested that a uniform roughness pad provided much higher removal rates due to the larger real contact area compared with a random roughness pad [14]. While it is very difficult to observe the in situ movements of active particles during polishing, the contact area of padwafer can be measured and has been considered as the real contact area in most cases.

Borucki has developed a tool to measure the real contact area of pad-wafer using the confocal microscopy and analyzed the contact image with digital image processing [15]. Philipossian et al. investigated the real contact area of a pad with a wafer as a function of the process/tool conditions, pad conditioner, polishing pad, and their combinations [16, 17]. They observed that a higher contact area of pad-wafer results in a higher material removal rate. Later, Lee measured the real contact area of the polishing pad using a home-built tool as a function of applied pressure and abrasive size and proposed a modified semi-empirical model to predict the removal rates of $\mathrm{SiO}_{2}$ films [18].

However, according to the classical contact mechanics, when the real contact area between pad and wafer increases, the real contact pressure decreases, leading to a decrease in the material removal rate. The opposite conclusion may be drawn from the properties of CMP consumables (slurry, films, polishing pad, conditioner, etc.), their characteristics, and CMP process conditions. More elaborate studies are needed to understand two opposing tendencies of material removal rates.

The classic equation for contact mechanics is Preston's equation [19] expressed as $\mathrm{MRR}=K P V$ where $\mathrm{MRR}$ is the material removal rate, $K$ is Preston's coefficient, $P$ is the applied pressure on the wafer surface, and $V$ is the relative velocity between the wafer and the polishing pad. Preston's equation was very useful in explaining most of the removal rates of dielectric materials in the early stages of CMP technology. However, it assumes a linear relationship between pressure $P$ and relative velocity $V$ and hence, is unable to explain nonlinear polishing behavior in actual CMP [20, 21]. Also, Preston's equation does not consider the properties of CMP consumables and their characteristics. As the CMP process becomes more and more complex, it has been difficult to predict CMP results and explain their mechanism by only Preston's equation. Today's contact mechanics for the CMP process not only consider the properties of CMP consumables and their characteristics but also simulate the fluid dynamics and process parameters, which provide more reliable information on the actual CMP mechanism [20]. 
Despite a number of such investigations, more fundamental studies are needed to investigate the in situ measurement of the real contact area of active particles at the slurry/pad-wafer interface during polishing, especially while considering $\mathrm{SiO}_{2}$ and $\mathrm{CeO}_{2}$ abrasive particles that have been typically used for CMP applications. Most previous models considered particle size and size distribution, where the particle shape was assumed to be spherical. Their type, hardness, shape, particle agglomeration, and chemical reactions between slurry and wafer surface have a significant influence on the material removal rates. These factors have to be taken into consideration in the mathematical model based solely on contact mechanics.

\section{Lubrication Models}

During polishing, CMP slurries are transported to grooved trenches, pores, and texture of a pad and improve hydrodynamic lubrication (Fig. 1). Sundararajan et al. proposed a lubrication model for slurry flow and a mass transport model for material removal by solving the Reynolds equation [22]. According to their numerical results, a $40-60 \mu \mathrm{m}$ slurry film between the wafer and the pad is expected at an applied pressure of $14 \mathrm{kPa}$ and a linear velocity of $1.3 \mathrm{~m} / \mathrm{s}$. Slurry film thickness decreased to the range of $15-30 \mu \mathrm{m}$ with higher applied pressure on the wafer. Lu et al. developed a dual emission ultraviolet (UV)-enhanced fluorescence technique using a dual-camera imaging system to measure the thickness of the slurry film between the pad and a BK-7 wafer and in situ monitored the average slurry film thickness during polishing [23].

Philipossian's group modified this tool with high spatial resolution images, high-intensity UV laser pulses, and enhanced fluorescence [Fig. 2(a)] [24]. They used two different fluorescent dyes that can be excited at different wavelengths and recorded the fluorescence intensities of the dye molecules in the slurry using two high-resolution cameras. The intensity ratio of light detected by each camera was converted to slurry film thickness after proper calibration [Fig. 2(a)] [23, 24]. They also studied the dependence of the slurry film thickness on the type of conditioner disk (design, kinematics, and pressure) [26] and pad properties (asperities, roughness, and groove) [25]. They measured the in situ coefficient of friction (COF) along with slurry film thickness. COF showed an inverse relationship with the average slurry film thickness. In thick and more viscous slurry films, the abrasive particles and the pad are less likely to interact with the wafer, resulting in a lower COF. The high viscosity of CMP slurries not only results in ineffective mass transport of reactants across the wafer and poor lubrication between the wafer and the pad but also restricts the movement of abrasive particles in the slurry.

The relationship between COF and the slurry film thickness is well explained by the Stribeck curve [Fig. 2(b)] [27, 28].
By plotting COF against the Hersey number [29], three distinct regions-boundary lubrication, mixed lubrication, and hydrodynamic lubrication-were identified. The Hersey number is given by $\eta V / P$ where $\eta$ is the slurry viscosity, $V$ is the relative velocity between pad and wafer, and $P$ is the pressure. Region I of this curve, known as boundary lubrication, shows relatively very high COF due to the direct contact between two solid surfaces. Region II is referred to as mixed lubrication where the wafer does not directly contact a pad because of the slurry film formed between the wafer and the pad. In Region III (hydrodynamic lubrication), the pad and the wafer are fully separated due to the relatively thick slurry film, resulting in a relatively low removal rate and $\mathrm{COF}$.

Mullany and Byrne experimentally and theoretically investigated the effect of slurry film thickness on COF during $\mathrm{Si}$ wafer polishing in the mixed lubrication region [30], showing that the removal rates of the Si wafer decrease with increasing Hersey number. However, the traditional Stribeck curve fails to explain the lubrication phenomena, while COF and downforce fluctuated significantly during polishing because of the so-called stick-slip phenomena [31, 32]. Han et al. developed a Stribeck+ curve involving instantaneous process valuables [31, 32] (measured shear force and downforce, calculated, and recorded COF), instead of using a constant of shear force and downforce throughout the polishing process, and successfully explained the Stribeck+ curves for $\mathrm{SiO}_{2}$ [31], $\mathrm{Cu}$ [32], and W CMP [32] using various combinations of CMP slurries and pads.

As mentioned earlier, viscosity is one of the key parameters that control the slurry film thickness and COF $[27,28]$. The Hersey number includes viscosity [29], and it was considered as a constant during polishing because most CMP slurries behave as Newtonian fluids displaying a viscosity that is independent of shear rate. However, Lortz et al. found that the viscosity of silica slurries was constant, like Newtonian fluids, only up to a shear rate of $10,000 \mathrm{~s}^{-1}$ and increased significantly for higher shear rates in the range of $200,000-300,000 \mathrm{~s}^{-1}$ [33]. Later, Crawford et al. developed a new methodology to in situ measure the rheological behavior of CMP slurries (in situ rheo-polishing setup) while polishing the wafer [34]. They observed a fivefold increase in the viscosity of the silica slurries in the presence of $0.15 \mathrm{M} \mathrm{KCl}$ with increasing shear rate $\left(\geq 10,000 \mathrm{~s}^{-1}\right)$. Silica particles can easily form a network structure and agglomerate in an aqueous medium under high shear rates due to their strong hydrogen bonding, triggering a shear-thickening effect $[35,36,37]$. Moreover, in some cases, the heat generated by the chemical and mechanical interactions at the wafer-pad-particle interface can have a significant influence on the slurry viscosity [38]. It usually decreases with increasing temperature. These changes in slurry viscosity should be reflected in the Stribeck curve. 

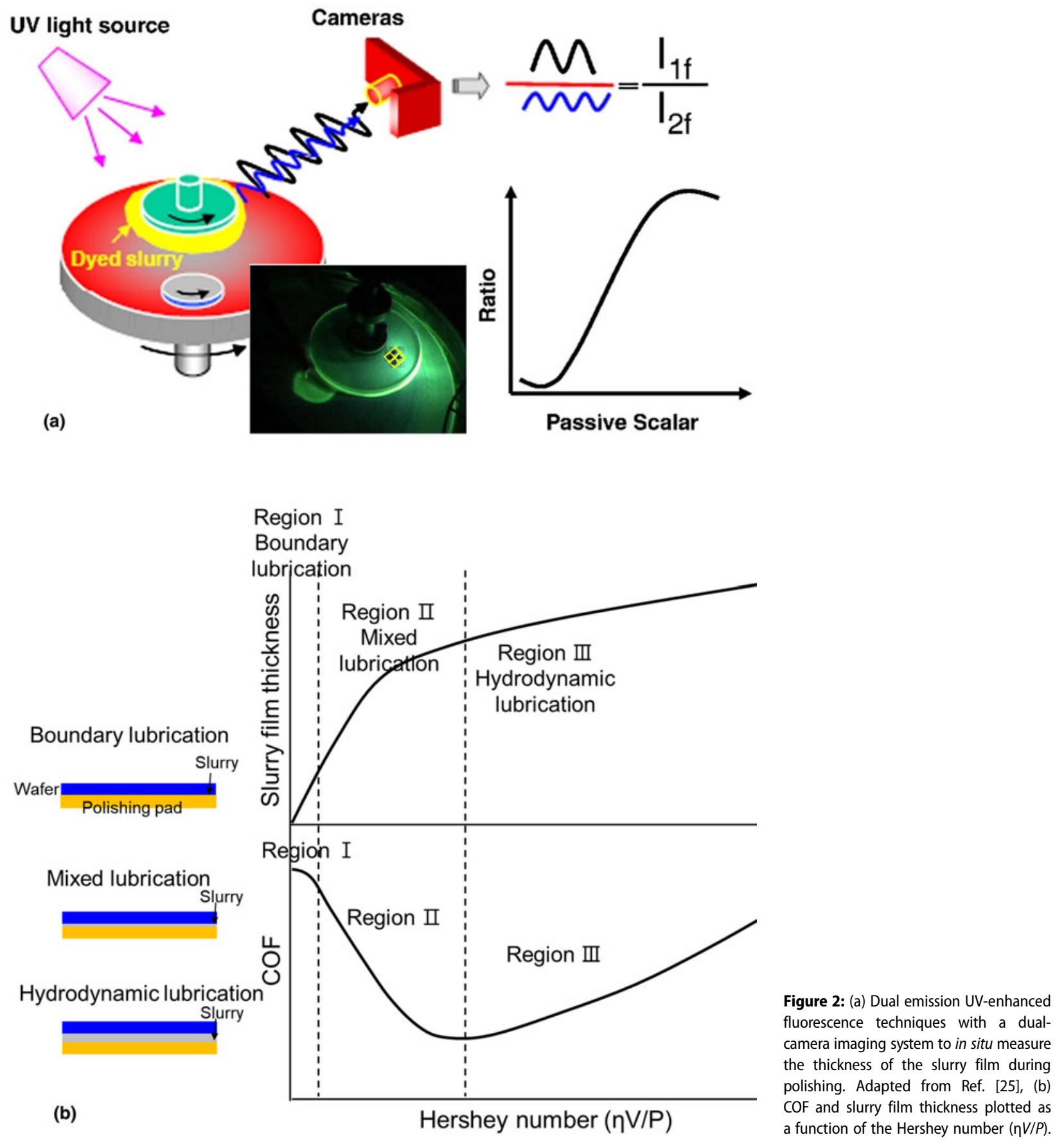

\section{Chemical Reactions Between Slurries and Dielectric Fillms}

The mechanical effect alone is not sufficient to explain the material removal. Thus, it is essential to understand the chemical reactions of slurry components with the wafer surface during polishing. Since a glass-polishing model was first developed in the 1980s by Brown et al. [39], various theories and concepts have been proposed to explain the removal of materials during polishing. Cook [40] investigated the removal rates of glass using the slurries composed of various abrasive particles (e.g., alumina, silica, ceria, zirconia, and titania) and found that the ceria particles can interact with the glass surface by forming the strong chemical bonds, which he called as "chemical-tooth" [Fig. 3(a)] [41]. In the aqueous medium, the glass surface reacts with water molecules and forms dissolved silanol $\left(\mathrm{Si}(\mathrm{OH})_{4}\right)$ as expressed by $\left(\mathrm{SiO}_{2}\right)_{x}+2 \mathrm{H}_{2} \mathrm{O} \leftrightarrow \quad\left(\mathrm{SiO}_{2}\right)_{x-1}+\mathrm{Si}(\mathrm{OH})_{4} \quad$ [42]. According to his model, $\mathrm{OH}$ groups on the ceria surface form strong $\mathrm{Ce}-\mathrm{O}-\mathrm{Si}$ bonds with silanol groups (-Si-OH/$\mathrm{Si}-\mathrm{O}^{-}$) on the glass surface during polishing [Fig. 3(a)]. The 

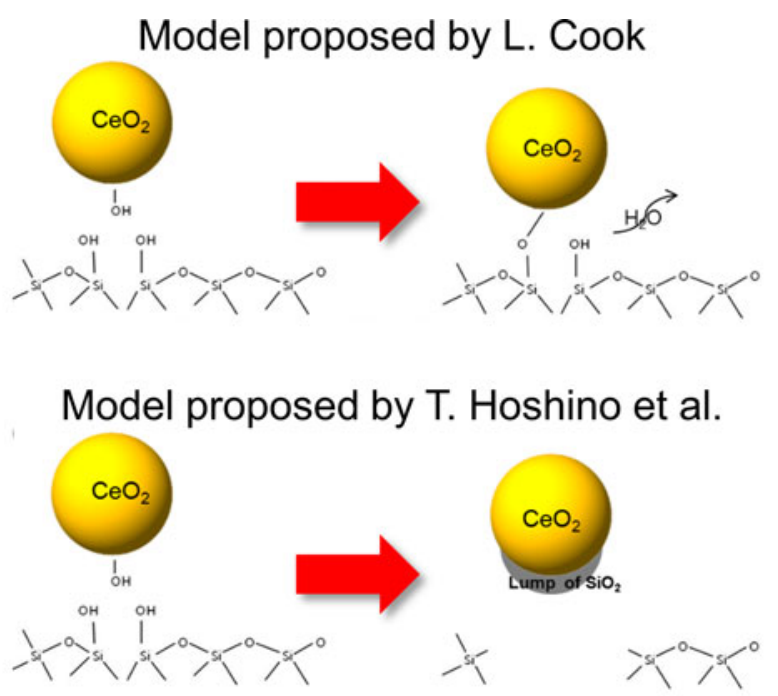

Model proposed by S.V. Babu et al.

(a)
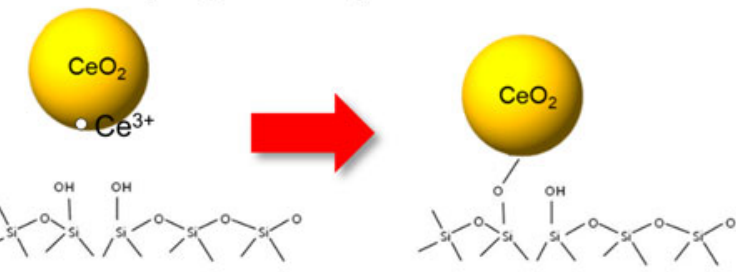

(b)

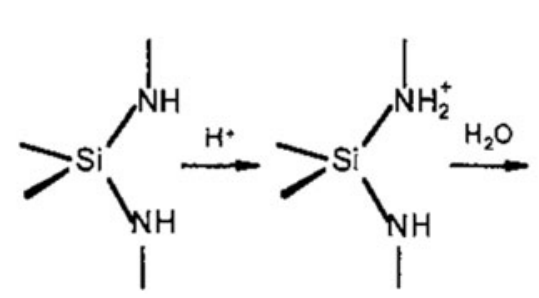<smiles>CN[Si](C)(C)[NH2+]C</smiles>

(c)

\section{Formation of passivating film}

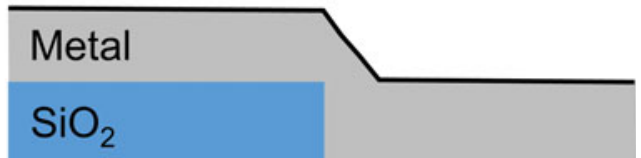

2. Removal of passivating film by mechanical action

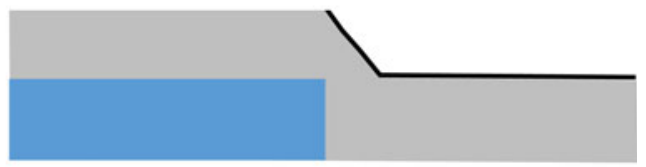

3. Dissolution of unprotected metal by chemical action;

Formation of passivating film

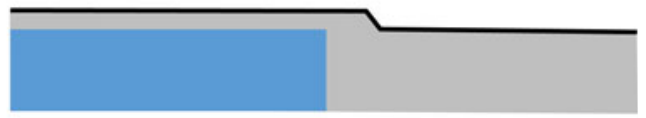

4. The continuous cycles of 2 and 3
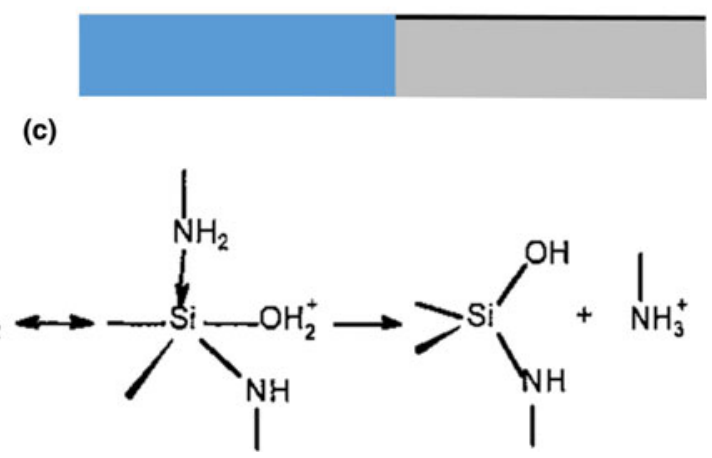

Figure 3: (a) Schematic illustration of $\mathrm{SiO}_{2}$ removal mechanisms and (b) $\mathrm{Si}_{3} \mathrm{~N}_{4}$ hydrolysis reaction mechanism. Adapted from Ref. [41]. (c) Schematic diagram of the material removal mechanism.

proposed chemical reaction is given by $-\mathrm{Ce}-\mathrm{OH}+-\mathrm{Si}-\mathrm{O}^{-} \leftrightarrow-$ $\mathrm{Ce}-\mathrm{O}-\mathrm{Si}-+\mathrm{OH}^{-}[42]$. The $\mathrm{Ce}-\mathrm{O}-\mathrm{Si}$ bonds are much stronger than $\mathrm{Si-O}-\mathrm{Si}$ bonds, which can be removed by both chemical reaction and mechanical abrasion during polishing. Later, Hoshino et al. [43] suggested that $\mathrm{SiO}_{2}$ surfaces are removed as the form of lumps of Ce-O-Si bonds based on Fourier transform infrared spectroscopy (FTIR) and inductively coupled plasma atomic emission spectroscopy analysis of the post-CMP slurries [Fig. 3(a)]. Babu's group studied the effects of the Ce oxidation state $\left(\mathrm{Ce}^{3+} / \mathrm{Ce}^{4+}\right)$ on the ceria surface on the interactions with $\mathrm{SiO}_{2}$ film using $\mathrm{UV} /$ visible spectroscopy and proposed that the surface $\mathrm{Ce}^{3+}$ sites stabilized by an oxygen vacancy are responsible for the high reactivity with $\mathrm{SiO}_{2}$ film [Fig. 3(a)] [44, 45]. They also showed that the reactivity of ceria particles can be lowered by the blocking of the active $\mathrm{Ce}^{3+}$ surface sites using various additives $[45,46]$. Based on adsorption isotherms of silicate ions, Seo et al. reported that ceria particles with higher surface $\mathrm{Ce}^{3+}$ concentrations have higher adsorption affinity with $\mathrm{SiO}_{2}$ films. Taking advantage of these reports, current research for achieving high $\mathrm{SiO}_{2}$ removal rates is aimed at preparing $\mathrm{Ce}^{3+}$ rich ceria particles through either impurity metal-doping or coating. It is well known that lanthanide ( $\mathrm{La}, \mathrm{Sm}, \mathrm{Gd}, \mathrm{Nd}$, and $\mathrm{Yb}$ ) doping can increase $\mathrm{Ce}^{3+}$ concentration at the ceria surface, thus enhancing the $\mathrm{SiO}_{2}$ removal rates $[47,48]$. Recently, Kim et al. have developed the core/shell type $\mathrm{Ce}^{3+}$ rich ceria abrasive for improved $\mathrm{SiO}_{2}$ removal rates [49]. Additionally, by coating the abrasives with a layer of different materials and chemical compositions, it is possible to change the physicochemical properties of the core abrasives and their reactivity with films [49].

$\mathrm{Si}_{3} \mathrm{~N}_{4}$ films can be easily oxidized to $\mathrm{SiO}_{2}$ at the surface when exposed to water or air, and then removed, as in the case of $\mathrm{SiO}_{2}$ films, by chemical reaction (the formation of Ce-O-Si bonds) as well as mechanical abrasion [50]. 
Figure 3(b) shows the overall hydrolysis of $\mathrm{Si}_{3} \mathrm{~N}_{4}$ film in an aqueous medium; an amine is liberated from $\mathrm{Si}_{3} \mathrm{~N}_{4}$ film through nucleophilic attack of water molecules, with the overall reaction described as $\mathrm{Si}_{3} \mathrm{~N}_{4}+6 \mathrm{H}_{2} \mathrm{O} \rightarrow 3 \mathrm{SiO}_{2}+4 \mathrm{NH}_{3}\left(\Delta G^{\circ}=\right.$ $-147 \mathrm{kcal} / \mathrm{mol}$ at $298 \mathrm{~K}$ ) [51]. The oxidized surface layer may be further hydrated as silicate species, which is written as $\equiv \mathrm{Si}-\mathrm{O}-\mathrm{Si} \equiv+\mathrm{H}_{2} \mathrm{O} \rightarrow \mathrm{Si}(\mathrm{OH})_{4}$. Laarz et al. [41] and Iler [52] showed that the rate of hydration is strongly affected by slurry $\mathrm{pH}$, surface area, the preparation method of film, temperature, etc. Also, Laarz et al. found that the oxidized surface layer on $\mathrm{Si}_{3} \mathrm{~N}_{4}$ film behaved like amorphous silica and determined the activation energy $(52 \mathrm{~kJ} / \mathrm{mol})$ for the hydrolysis of the amorphous surface layer [41]. The conversion process/reaction of $\mathrm{Si}_{3} \mathrm{~N}_{4}$ film is important in determining the removal mechanism of $\mathrm{Si}_{3} \mathrm{~N}_{4}$, and its removal rate [50]. Babu and co-workers investigated various amino acids (alpha, cyclic, and aliphatic) that can be preferentially adsorbed onto $\mathrm{Si}_{3} \mathrm{~N}_{4}$ film, inhibiting its hydrolysis to $\mathrm{SiO}_{2}[53,54]$. The details of the mechanism of $\mathrm{Si}_{3} \mathrm{~N}_{4}$ removal rate suppression will be discussed in detail. Later, Alety et al. showed that the removal rate of $\mathrm{Si}_{3} \mathrm{~N}_{4}$ film in the presence of $0.1 \mathrm{wt} \%$ ceria particles $+2.3 \mathrm{mM} \mathrm{Ce}\left(\mathrm{NO}_{3}\right)_{3}$ at $\mathrm{pH} 4$ was $\sim 300 \mathrm{~nm} / \mathrm{min}$, whereas it is $\sim 10 \mathrm{~nm} / \mathrm{min}$ with only ceria particle [55]. X-ray photoelectron spectroscopy of the polished $\mathrm{Si}_{3} \mathrm{~N}_{4}$ films is similar to that of an as-received silicon oxynitride film which led them to suggest that, in the presence of $\mathrm{Ce}^{3+}$, the surface of $\mathrm{Si}_{3} \mathrm{~N}_{4}$ film can be converted to silicon oxynitride which was then polished at a high rate [55].

\section{Chemical Reactions Between Slurries and Metal Films}

Unlike the removal mechanisms of $\mathrm{SiO}_{2}$ and $\mathrm{Si}_{3} \mathrm{~N}_{4}$ films, metal films (W, $\mathrm{Cu}, \mathrm{Ru}, \mathrm{Ta}$, and some of their alloys) are very difficult to be polished by only abrasive particles due to their inertness and hardness [1]. Kaufman et al. first proposed the polishing mechanism of W films when the slurry was composed of ferricyanide, hydrogen phosphate, and ethylenediamine [56]. A "soft" passivating layer forms on the $\mathrm{W}$ film in the presence of ferricyanide, $\left[\mathrm{Fe}(\mathrm{CN})_{6}\right]^{3-}$, as an oxidizer, which can be written as $\mathrm{W}+6\left[\mathrm{Fe}(\mathrm{CN})_{6}\right]^{3-}+4 \mathrm{H}_{2} \mathrm{O} \rightarrow \mathrm{WO}_{4}^{2-}+6\left[\mathrm{Fe}(\mathrm{CN})_{6}\right]^{4-}+$ $8 \mathrm{H}^{+}$[56]. They proposed that the "soft" passivation layer can be easily removed by mechanical abrasion, and the fresh metal is exposed to the oxidizer again, which is subsequently passivated and then removed [56]. Paul and co-workers proposed chemical kinetics of $\mathrm{W}$ [57] and $\mathrm{Cu}$ films [58] as a function of oxidizer, chelating agent, inhibitor, and mechanical contribution with a mechanical abrasion process. The passivation layer $\mathrm{MC}^{\star}$ forms on the metal film $\mathrm{M}$ in the presence of chemical component $\mathrm{C}$ by the chemical reaction; $\mathrm{M}+$ $\mathrm{C} \leftrightarrow \mathrm{MC}^{*}$. The passivation layer can be partially dissolved in the slurries $\left(\mathrm{M}_{\mathrm{bulk}}+\mathrm{MC}^{\star} \rightarrow \mathrm{MC}_{\mathrm{aq}}+\mathrm{M}\right)$ or removed by mechanical abrasion $\left(\mathrm{MC}^{\star}+\mathrm{A} \rightarrow \mathrm{MC}-\mathrm{A}+\mathrm{M}\right)$ where $\mathrm{MC}_{\mathrm{aq}}$ is the dissolved species of $\mathrm{MC}, \mathrm{A}$ is the abrasive particle, and $\mathrm{MC}-\mathrm{A}$ is the leaving material from the metal surface. Kinetics of competitive reactions of film formation $r_{\mathrm{f}}=k_{\mathrm{f}} N_{\mathrm{M}}[\mathrm{C}]$ by oxidizer, film dissolution $r_{\mathrm{d}}=k_{\mathrm{d}} N_{\mathrm{MC}^{*}}$ by chelating agent, and mechanical abrasion $r_{\mathrm{M}}=k_{\mathrm{M}}\left(N_{\mathrm{a}} / A\right) N_{\mathrm{MC}}$ by particles determine the removal rates of the metal film where $k_{\mathrm{f}}, k_{\mathrm{d}}$, and $k_{\mathrm{M}}$ are the rate constants for the reactions, $N_{\mathrm{M}}$ and $N_{\mathrm{MC}^{*}}$ are the number of $\mathrm{M}$ and $M C^{\star}$ sites on the metal $M$ surface, [C] is the concentration of the chemical component $\mathrm{C}$ in the slurry and $N_{\mathrm{a}}$ is the number of the active abrasive particles per unit area $A$ of the metal surface. Later, Paul and Vacassy extended their model to the systems with inhibitor by combining the reaction and kinetics described above [59]. The mechanism of this process is used to explain other metal CMP processes, and the possible chemical reactions at the metal surface are summarized below $[60,61]$.

Metal + Oxidizer $\rightarrow$ Metal oxide + partially dissolved

Metal/Metal oxide + Chelating agent

$\rightarrow$ Formation of the insoluble passivating layer or

Dissolved complex ion

Metal + Inhibitor $\rightarrow$ Formation of the insoluble passivatinglayer

Metal $+\mathrm{H}^{+} / \mathrm{OH}^{-} \rightarrow$ Dissolved as $\mathrm{M}^{n+}$

Various oxidizers such as $\mathrm{H}_{2} \mathrm{O}_{2}, \mathrm{KIO}_{3}, \mathrm{Fe}\left(\mathrm{NO}_{3}\right)_{3}, \mathrm{~K}_{2} \mathrm{~S}_{2} \mathrm{O}_{8}$, and their mixtures have been investigated [56, 62, 63, 64]. $\mathrm{H}_{2} \mathrm{O}_{2}$ has been widely used as an oxidizer for many metal CMP slurries due to its low cost and powerful oxidizing capability $[65,66]$. When both ferric ions and $\mathrm{H}_{2} \mathrm{O}_{2}$ are present, ferric ions can lead to the decomposition of $\mathrm{H}_{2} \mathrm{O}_{2}$ into the free hydroxyl radicals $(\cdot \mathrm{OH})$, powerful oxidants, through the Fenton reaction. These radicals can rapidly form a "soft" passivation layer on the metal film, resulting in high MRR and improved topography of metal film $[62,67]$. Chelating agents such as glycine and citric acid that form a complex with metal ions can also help in the material removal by forming either insoluble or soluble surface complexes on the metal surface $[68,69]$. However, corrosion defects such as pitting, cracking, and excessive etching will inevitably occur when the metal films are exposed to corrosive reagents [70]. Organic inhibitors containing heteroatoms such as $\mathrm{O}, \mathrm{N}$, and $\mathrm{S}$ have been used to inhibit the corrosion of a metal. They can be effectively chemisorbed on the metal surface through the lone pair of electrons on these heteroatoms. Azole derivatives such as benzotriazole (BTAH, often referred to simply as BTA) and 1,2,4-triazole (TAZ) have been used as a corrosion inhibitor for the metal film during polishing, as discussed later in the section on the inhibitor. 
The mechanism of the metal CMP process involving the continuous cycles of the formation of a passivating layer on the metal film by oxidizer and inhibitor, removal of the passivating layer by the mechanical action, dissolution by chemical reactions, and re-passivation of the metal film is shown in Fig. 3(c).

\section{Electrochemical Reactions}

Metal CMP is governed by electrochemical behaviors of metal films in the presence of oxidizer, complexing agents, and corrosion inhibitors. When exposed to slurry components, the metal film is oxidized and loses electron, forming metal cation (anodic reaction, $\mathrm{M}=\mathrm{M}^{+}+\mathrm{e}^{-}$) and the corresponding cathodic reduction reaction receives electrons $\left(\mathrm{O}_{2}+4 \mathrm{H}^{+}+4 \mathrm{e}^{-}=2 \mathrm{H}_{2} \mathrm{O}\right)$. Oxidation/dissolution and passivation of metal films occur at the open circuit potential (OCP) of each system [71]. The OCP transients stabilize when the anodic and cathodic sites arrive at a saturation point, and the OCPs and $\mathrm{pH}$ values of the slurries are used to predict the thermodynamically stable chemical compounds or species of a given metal film in the slurry components according to Pourbaix diagrams (or E-pH diagrams). The general $E$-pH-dependent reaction at $25^{\circ} \mathrm{C}$ can be expressed as $a(\mathrm{Ox})+m \mathrm{H}^{+}+n \mathrm{e}^{-} \leftrightarrow b(\mathrm{Rd})+c \mathrm{H}_{2} \mathrm{O}$ where $a, m, b$, and $c$ are the number of molecules, $n$ is the number of electrons involved in the reaction, and Ox and Rd denote the oxidized and reduced species of the active redox couple, respectively. E-pH diagrams were constructed by using the Nernst equation and appropriate equilibrium constant expressions [72]: $E=E^{0}-(2.303 R T m) /(n F) \mathrm{pH}+(2.303 R T / n F)$ $\log \left(C_{\mathrm{Ox}}{ }^{a} / C_{\mathrm{Rd}}{ }^{b}\right)$ where $E$ is the actual cell potential at nonstandard condition, $E^{0}$ is the standard reduction potential, $R$ is the gas constant, $T$ is the absolute temperature, $F$ is the Faraday constant, and $C_{\mathrm{Ox}}$ and $C_{\mathrm{Rd}}$ represent the concentration of the species $\mathrm{Ox}$ and $\mathrm{Rd}$ involved in the $E$-pH-dependent reactions. Pourbaix diagrams for various metal-slurry systems for CMP applications have been developed [73].

A goal of metal CMP is to uniformly polish the interconnection materials $(\mathrm{Cu}, \mathrm{Co}, \mathrm{Al}, \mathrm{W}$, etc.) and the barrier materials ( $\mathrm{Ta}, \mathrm{TaN}, \mathrm{Ti}, \mathrm{TiN}, \mathrm{Co}, \mathrm{Ru}$, etc.) with the desired removal rates and selectivity while minimizing CMP-related defects such as localized pitting, dissolution, and galvanic corrosion, and stop on the oxide layer. The interconnection materials and the barrier materials are in physical contact, and the polishing slurry provides a conductive pathway between them. According to mixed potential theory, two metals with different potentials are polarized until they reach the same potential, forming a galvanic couple. The more noble metal becomes a cathode for the oxygen reduction reaction, and the less noble metal is corroded more rapidly through dissolution/oxidation while supplying electrons to the cathode, resulting in the preferential dissolution/corrosion of either interconnection material or barrier material by galvanic corrosion (Fig. 4) [73]. Corrosion parameters $\left(E_{\text {corr }}, I_{\text {corr }}, E_{\mathrm{g}}\right.$, and $\left.I_{\mathrm{g}}\right)$ obtained from Tafel extrapolations provide useful information on the oxidation-reduction reactions of metal films in the slurry. Chemical additives induce the shifts in the $E_{\text {corr }}$ and the $I_{\text {corr }}$ by the complex mechanism. An upward shift of Tafel curves can occur due to anodic
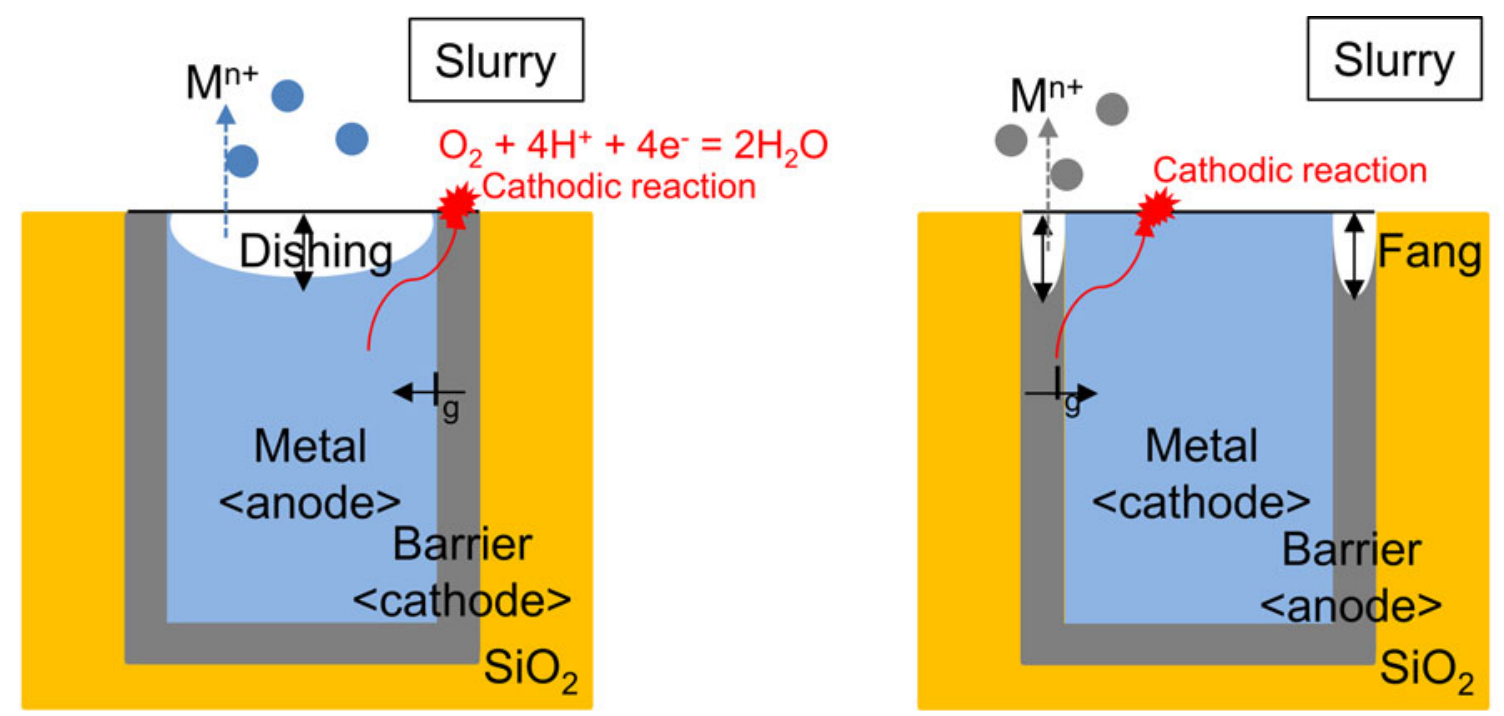

\section{Potential: Metal > Barrier}

\section{Potential: Metal < Barrier}

Figure 4: Galvanic corrosion of metal-barrier couple due to their potential difference. The preferential dissolution/corrosion of interconnection material and barrier material leads to the dishing and the fang, respectively. 
corrosion/passivation or cathodic stimulation. A downward shift in Tafel plots is observed with the addition of the cathodic inhibitor by retarding the rate of oxygen transfer to the cathodic sites on the metal films. It is necessary to minimize the potential difference of the metal/barrier couple with low corrosion currents through a careful selection of oxidizer, complexing agent, corrosion inhibitor, and the slurry $\mathrm{pH}$. Cu metal and $\mathrm{Ru}$ barrier act as the anode and cathode of the galvanic couple, respectively. Galvanic corrosion of $\mathrm{Cu}-\mathrm{Ru}$ couple needs to be controlled through the selective prevention of cathodic reactions on $\mathrm{Ru}$ film, and/or increasing the potential of $\mathrm{Cu}$ by preferential anodic inhibition of $\mathrm{Cu}$ films. Peethala et al. [74] proposed a silica-based slurry containing $5 \mathrm{mM}$ BTA and $7 \mathrm{mM}$ ascorbic acid at $\mathrm{pH} 9$ in the presence of $\mathrm{KIO}_{4}$, as an oxidizer as selective anodic and cathodic corrosion inhibitors for $\mathrm{Cu}$ and $\mathrm{Ru}$ films, respectively. The mixed corrosion inhibitor system not only minimized the galvanic corrosion of $\mathrm{Cu}-\mathrm{Ru}$ couple but also achieved a reasonable removal rate selectivity of $\mathrm{Cu}$ and $\mathrm{Ru}$ films. Chockalingam et al. [75] showed that $2 \mathrm{mM}$ BTA and $3 \mathrm{mM}$ sucrose in the presence of $\mathrm{KIO}_{4}$ at $\mathrm{pH} 10$ were able to suppress both anodic and cathodic reaction of $\mathrm{Cu}$ and $\mathrm{Mn}$ films by forming a passivation layer on the films, leading to minimizing the individual corrosion of films as well as their galvanic corrosion. Jiang et al. [76] showed that the combination of BTA and Pluronic ${ }^{\oplus} \mathrm{P} 103$ in the presence of $\mathrm{KIO}_{4}$ at $\mathrm{pH}$ 9.5 formed a compact passivation layer, composed of Pluronic ${ }^{\circ}$ $\mathrm{P} 103$ adsorbed on the $\mathrm{Cu}-\mathrm{BTA}$ complex, resulting in the desired $\mathrm{Cu}$ removal rate $\sim 200 \AA / \mathrm{min}$ with the negligible dissolution and excellent surface quality. This slurry composition was also very effective in the control of the removal rates of $\mathrm{Cu}, \mathrm{Ru}$, and low- $k$ dielectric films and their selectivity, but the galvanic corrosion of $\mathrm{Cu} / \mathrm{Ru}$ couple needed to be reduced for improved surface quality when $\mathrm{Ru}$ is used as the barrier layer.

\section{Adsorption Behavior and Mechanism of Slurry Components on Abrasive Particles and Films}

Organic compounds such as dispersants and passivation agents in CMP slurries have been used to stabilize the slurry and achieve the selective removal of the materials during polishing, respectively. The most common functional groups of dispersants and passivation agents used in CMP slurries are carboxylic acids $(\mathrm{COOH})$ and amines $\left(\mathrm{NH}_{2}\right)$. Chemical adsorption between the adsorbate and the surface occurs through various interactions such as electrostatic, hydrogen bonding, and hydrophobic interactions [77]. Electrostatic interaction is relevant for ionic species and involves the attractive electrostatic forces between opposite charges. Hydrogen bonding occurs when electronegative atoms such as $\mathrm{O}, \mathrm{S}$, or $\mathrm{N}$ approach a hydrogen atom bound to another electronegative atom. It is possible for organic compounds containing electronegative groups such as $-\mathrm{OH},-\mathrm{O}-,-\mathrm{NH}$, and $-\mathrm{NH}_{2}$ to adsorb onto $-\mathrm{OH}$ groups of the hydrated surface of particles and films through hydrogen bonding.

\section{Adsorption Behavior and Mechanism of Dispersants on Abrasive Particles}

Poly(acrylic acid) (PAA) and copolymers, anionic polymers, have been widely used as dispersants for ceria slurries [78, 79]. When added to the slurry, the $\mathrm{COOH}$ groups of PAA are deprotonated to negatively charged $\mathrm{COO}^{-}$groups above its pKa of 4.5 and adsorb on the highly positively charged ceria surface $\left(\mathrm{OH}_{2}^{+}\right.$sites $)$via electrostatic attractive interaction and hydrogen bonding [Fig. 5(a)] [80], which increases
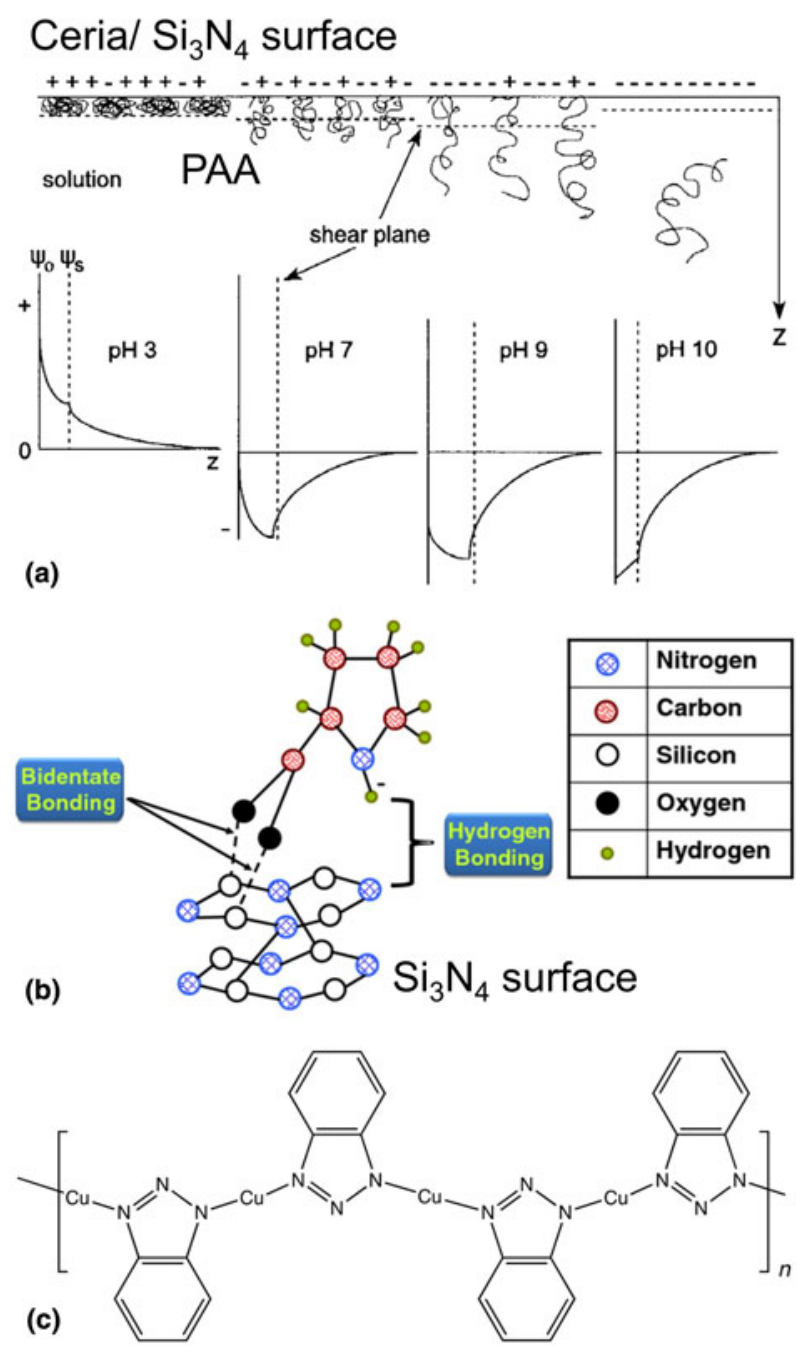

Figure 5: (a) Schematic illustration depicting PAA interactions at the ceria/ $\mathrm{Si}_{3} \mathrm{~N}_{4}$-slurry interface as a function of $\mathrm{pH}$ and corresponding potential-distance diagrams. Adapted from Ref. [80]. (b) Bond formation between proline and silicon nitride surface. Adapted from Ref. [53]. (c) Cu-BTA complex chemisorbed on the Cu surface. 
electrostatic repulsion as well as steric hindrance between the ceria particles. PAA coated ceria particles are known to undergo transitions of bridging agglomeration-stable-flocculation depending on their physicochemical conditions such as $\mathrm{pH}$, ionic strength, temperature, and concentration [81]. Citric acid and PAA have been used to disperse alumina abrasives $[82,83]$. Song et al. suggested that the mixed dispersant system of citric acid and PAA was very useful for the stable dispersion of alumina abrasives, leading to higher $\mathrm{Cu}$ removal rates by suppressing the hydration of alumina abrasive [84].

Ionic surfactants such as ammonium lauryl sulfate (ALS) and cetyl trimethyl ammonium bromide (CTAB) have been used in the formation of ceria slurries reported by Dylla-Spears et al. [85]. They suggested that the formation of charged micelles above the critical micelle concentration may electro-sterically hinder the agglomeration of ceria particles, which is called the "charged micelle halo" stabilization mechanism [85].

Tseng et al. [86] reported that the silica abrasives in the presence of methyl methacrylate were stabilized by either electrostatic barriers or steric barriers between the particles, resulting in an excellent uniformity of the oxide thickness across the wafer. Pan et al. [87] suggested that sodium dodecyl sulfate (SDS), an anionic surfactant, could improve the dispersion stability of silica-based slurries for Cu CMP. Indeed, free SDS surfactants remaining in the slurry were very useful to suppress $\mathrm{Cu}$ corrosion and lead to good surface quality. Polyethylenimine (PEI) and CTAB composed of the amine groups were used to disperse silica abrasive particles also [88, 89]. They can be adsorbed onto the negatively charged silica through electrostatic attractive interaction as well as hydrogen bonding and prevent agglomeration. However, the adsorbed layer on the particle surface results in the low material removal rate by preventing particles from direct contact with the materials to be polished.

\section{Adsorption Behavior and Mechanism of Passivation Agents on Films}

PAA, when used as a passivation agent during polishing, can be preferentially adsorbed on the positively charged $\mathrm{Si}_{3} \mathrm{~N}_{4}$ film ( $\left.\mathrm{pH}_{\text {IEP }} \sim 5-7\right)$ through electrostatic attraction [Fig. 5(a)] [80], suppressing the removal of $\mathrm{Si}_{3} \mathrm{~N}_{4}$ film and yielding high selectivity [90]. Kim et al. reported that PAA has almost ten times higher affinity with $\mathrm{Si}_{3} \mathrm{~N}_{4}$ at $\mathrm{pH}$ 5-9 than with $\mathrm{SiO}_{2}$ [91]. The preferential adsorption of PAA on $\mathrm{Si}_{3} \mathrm{~N}_{4}$ films was confirmed by attenuated total reflection (ATR)-FTIR spectra of $\mathrm{SiO}_{2}$ and $\mathrm{Si}_{3} \mathrm{~N}_{4}$ surfaces treated with PAA. Kim et al. measured the thickness of adsorbed PAA on the $\mathrm{Si}_{3} \mathrm{~N}_{4}$ film using atomic force microscopy as a function of ionic strength [92]. The thickness of the adsorbed PAA layers on the $\mathrm{Si}_{3} \mathrm{~N}_{4}$ film was
5.2, 4.5, and $3.8 \mathrm{~nm}$ with $0,0.2$, and $0.4 \mathrm{M} \mathrm{KNO}_{3}$, respectively. In some case, if the attractive forces such as hydrogen bonding are more dominant than the electrostatic repulsion between PAA and $\mathrm{SiO}_{2}$ film, it is possible for the adsorption of PAA to occur on the $\mathrm{SiO}_{2}$ film [93].

Park et al. investigated the effect of adding both PAA and poly(vinyl pyrrolidone) (PVP) to ceria based slurries to achieve the multi-selectivity between $\mathrm{SiO}_{2}, \mathrm{Si}_{3} \mathrm{~N}_{4}$, and poly-Si films and showed high selectivity $\left(\sim 65: \sim 15: 1\right.$ for $\mathrm{SiO}_{2}: \mathrm{Si}_{3} \mathrm{~N}_{4}$ :poly-Si films) in the presence of $0.05 \mathrm{wt} \%$ PAA and $0.2 \mathrm{wt} \%$ PVP in the $\mathrm{pH}$ range of 6.0-6.5 [94]. Seo et al. proposed the PAA-poly(ethylene glycol) (PEG) "interpolymer complexes" as a passivation agent for high selectivity with low dishing and reported that the cross-linked network structure of interpolymer complexes significantly reduced dishing by preventing abrasives from polishing oxide in the trenches [95].

America and Babu [53] proposed that the $\mathrm{COOH}$ group of the amino acid proline can form a bidentate interaction with the $\mathrm{Si}-\mathrm{OH}$ group on $\mathrm{Si}_{3} \mathrm{~N}_{4}$ film along with $\mathrm{H}$-bonding between the amino acid and $\mathrm{Si}_{3} \mathrm{~N}_{4}$ film [Fig. 5(b)]. The bidentate bonding is observed where two or more hydrogen bonds are formed with a base or base pair. More generally, many amino acids were able to suppress the removal rates of $\mathrm{Si}_{3} \mathrm{~N}_{4}$ film through the prevention of the hydrolysis of the $\mathrm{Si}_{3} \mathrm{~N}_{4}$ as long as there are available in sufficient amounts during polishing. Since amino acids can also be adsorbed on the abrasive particles, free amino acids remaining in the solution must be of sufficient quantity for effective suppression of $\mathrm{Si}_{3} \mathrm{~N}_{4}$ film removal rates. Since these amino acids are sensitive to a change of $\mathrm{pH}$, the slurry $\mathrm{pH}$ needs to be optimized based on their $\mathrm{pKa}$ values $[54,96]$.

Hydrophobic interaction involves the adsorption of the nonpolar groups of organic molecules such as a hydrocarbon backbone $\left(-\mathrm{CH}_{2}-\mathrm{CH}_{2}-\right)$ onto hydrophobic surfaces. Penta et al. [97] investigated four different anionic surfactants and showed that these negatively charged surfactants could preferentially adsorb on positively charged $\mathrm{Si}_{3} \mathrm{~N}_{4}$ film via electrostatic interactions. A monolayer of anionic surfactants is formed by electrostatic attraction between surfactants and $\mathrm{Si}_{3} \mathrm{~N}_{4}$, followed by a secondary layer by hydrophobic interaction between the hydrophobic tails of anionic surfactants, suppressing $\mathrm{Si}_{3} \mathrm{~N}_{4}$ removal rates. Nonionic surfactants that can preferentially adsorb on the hydrophobic poly-Si surface via hydrophobic interaction for preferential removal of $\mathrm{SiO}_{2}$ over poly-Si were studied by Lee et al. [98], and there was a strong dependence of the selectivity on the hydrophilic-lipophilic balance value and molecular weight of nonionic surfactants.

Metal films are prone to dissolve when exposed to reactive components in the slurry, and corrosion inhibitors are added to prevent the corrosion defects such as pitting, cracking, and excessive etching during polishing. Most of the inhibitors 
studied for CMP applications are organic compounds containing $\mathrm{N}, \mathrm{S}$, and $\mathrm{O}$ atoms [99]. A metal cation can interact with aromatic rings containing conjugated bonds, $\pi$ electrons, and lone-pair electrons from heteroatoms $(\mathrm{O}, \mathrm{N}$, or $\mathrm{S})$. The efficiency of heteroatoms in the inhibitor molecules increases in the order of $\mathrm{O}<\mathrm{N}<\mathrm{S}$. The corrosion inhibition of metal films involves either physisorption or chemisorption of the inhibitor on the metal surface. Electrostatic attractive force between the charged inhibitor molecules and the charged metal surface leads to physisorption. Chemisorption is due to the interaction between unshared electron pairs of the heteroatom and metal atoms to form coordinate covalent bonds. The standard adsorption free energy $\left(\Delta G_{\text {ads }}^{\circ}\right)$ can provide insight into the mechanism of corrosion inhibition. The $\Delta G^{\circ}$ ads values above $-20 \mathrm{~kJ} / \mathrm{mol}$ and below $-40 \mathrm{~kJ} / \mathrm{mol}$ correspond to the physisorption and chemisorption process, respectively. Anodic inhibitors reduce the corrosion potential of metal films by oxidizing a surface layer and forming a thin passivating film on the metal. Cathodic inhibitors suppress the cathodic reaction $\left(\mathrm{O}_{2}+2 \mathrm{H}_{2} \mathrm{O}+4 \mathrm{e}^{-}=4 \mathrm{OH}^{-}\right)$by retarding the rate of oxygen transfer to the cathodic sites on the metal surface. Molecules with aliphatic chains are effective in suppressing the corrosion of metal films due to the attraction of nonpolar hydrophobic parts of inhibitor and polar medium [99]. The hydrophobic parts of inhibitors form a protective layer on the metal surface. Their size and molecular weight have an impact on the inhibition efficiency [100]. Larger the molecule, higher inhibition efficiency: $\mathrm{R}_{3} \mathrm{~N}>\mathrm{R}_{2} \mathrm{NH}>\mathrm{RNH}_{2}$, where $\mathrm{R}$ is a hydrocarbon chain.

Azole derivatives such as BTA and TAZ have been widely used as corrosion inhibitors for metal films during polishing [Fig. 5(c)] [101, 102, 103]. BTA can be adsorbed onto a $\mathrm{Cu}$ film by the coordination of the lone-pair electrons of the $\mathrm{N}$ atoms in BTA [Fig. 5(c)] with the $\mathrm{Cu}$ atoms on the surface $[104,105]$. Xue et al. suggested that BTAH reacts with metallic $\mathrm{Cu}$ much faster than on $\mathrm{Cu}$ oxide and forms a polymeric passivating layer for $\mathrm{Cu}$ film than for copper oxides [105]. Also, they showed that $\mathrm{Cu}$ could be oxidized to $\mathrm{Cu}(\mathrm{I})$ in the presence of dissolved oxygen and form a $\mathrm{Cu}(\mathrm{I}) \mathrm{BTA}$ complex with BTA: $4 \mathrm{Cu}+4 \mathrm{BTAH}+2 \mathrm{O}_{2} \leftrightarrow \mathrm{Cu}(\mathrm{I}) \mathrm{BTA}+2 \mathrm{H}_{2} \mathrm{O}$ [105].

Although surfactants such as CTAB [106], ammonium dodecyl sulfate [65], and potassium oleate [107] were also investigated as corrosion inhibitors, BTA is the most common corrosion inhibitor used in CMP slurries. In any case, all these inhibitors can complex with metal ions and remain stable on the metal film as undesirable organic residues [108], and it has been very difficult to remove these organic residues during post-CMP cleaning $[109,110]$. So, post-CMP cleaning has become essential to remove these organic residues from the films while minimizing other defects (e.g., residual particles, foreign materials, scratches, and corrosion). Figure 6 shows a summary of the adsorption of organic compounds such as dispersants, passivation agents, and inhibitors on the abrasive particles and the wafer surfaces in the CMP system.

\section{Temperature Effects}

The major sources of thermal heating during polishing are frictional dissipation and the chemical reactions of slurry components at the slurry/pad-wafer interface, leading to a change in temperature. Various thermal models of the polishing process based on kinematics [111], energy generation [112], and a combination of frictional heating and chemical reactivity [113] were proposed. Infrared thermography was used to characterize the impact of thermal effects on pad life, polishing rate, and non-uniformity during polishing. Wang et al. [114] reported that the exothermic reaction between the metal surface and the oxidizers in the acidic environment could increase the temperature up to $75^{\circ} \mathrm{C}$ during polishing depending on CMP conditions, which was lowered by the slurry flow. White et al. [112] proposed an energy flow mechanism into and out of the process, and an energy balance was used to predict an increase in the slurry temperature during polishing. They suggested that the heat is generated by the mechanical abrasion and the chemical reactions associated with the slurry chemistry and enthalpy is primarily transferred into the polishing pad, and then, most of the heat will be removed by convection of slurry flow, conduction, and radiation to the environment [112]. Oh and Seok [113] suggested that frictional heat generated during polishing is transferred to the wafer, pad, and slurry, and plays a key role in accelerating the chemical reactions at the slurry/pad-wafer interface. Their theoretical results showed that the temperature variation of the wafer, pad, and slurry has an influence on material removal rates. Indeed, the temperature rise of the CMP process not only has an effect on the slurry viscosity, $\mathrm{pH}$, particle size, and zetapotential $[30,115,116]$ but also leads to delamination or peeloff of the metal films $[70,117]$. For example, a $5{ }^{\circ} \mathrm{C}$ change in temperature can lead to a $10 \%$ drop in slurry viscosity [30]. Kakireddy reported that thin $\mathrm{Cu}$ film peeled off during polishing at higher temperatures due to lower adhesion and mechanical strength [117].

\section{The Effect of Temperature Changes on the Chemical and Mechanical Reactions during Polishing}

As stated earlier, the scope of this review paper is limited to CMP slurries and related to chemical and mechanical aspects of the CMP process, although other CMP consumables such as pad, pad conditioner, wafer carrier, and retaining ring and CMP conditions need to be considered. Figure 7 shows a 
Figure 6: An overview of the adsorption of organic compounds such as dispersants, passivation agents, and inhibitors on the abrasive particles and the wafer surfaces.
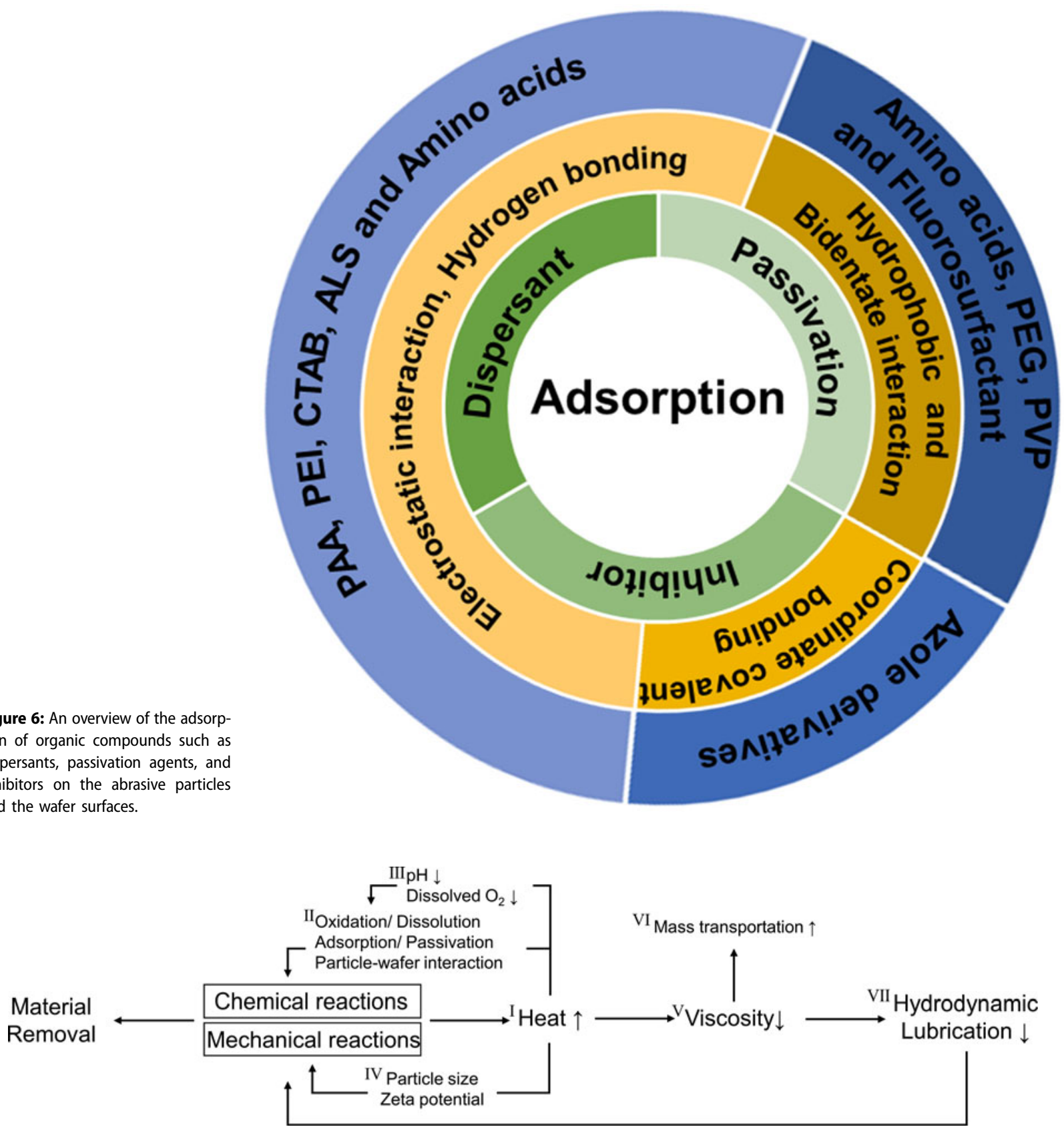

Figure 7: Schematic representation of the complex interactions between chemical and mechanical effects in the actual CMP system.

simplified scheme of the complex interactions between chemical and mechanical aspects of the CMP process discussed in this review paper. It is important to emphasize that Fig. 7 helps in understanding the complex interactions between chemical and mechanical effects that occur at the pad-waferparticle interface during polishing.

(i) Case I: The materials or wafers are removed by the synergetic effects of chemical and mechanical interaction during polishing. The energy dissipation due to frictional heating is responsible for increases in the temperaturesd during polishing. The average temperature of slurry increased up to $75^{\circ} \mathrm{C}$ during the W CMP process, and Wang et al. suggested that the oxidation of $\mathrm{W}$ film causes the temperature increase because this reaction is exothermic [114]. Also, in some cases, chemical reactions like the Fenton reaction are spontaneous exothermic processes, contribute to temperature increase during polishing.

(ii) Case II: As is well known, with increasing temperature of the system, the rates of chemical reactions increase [118], leading to higher removal rates $[116,119,120]$. Kim et al. 
reported that the slurries at higher temperatures lead to the formation of the soft layers on the surface of $\mathrm{SiO}_{2}$ film, and this layer could be easily removed by mechanical abrasion [116, 119]. Mudhivarthi et al. found that an increase in the surface oxidation/dissolution rate of $\mathrm{Cu}$ film, resulting in higher $\mathrm{Cu}$ removal rates [120]. The corrosion rate of metal films increases with increasing the temperature, leading to a decrease in the inhibition efficiency.

Moreover, an increase in temperature not only leads to changes in the polymer chain conformation (coiled transformed to stretched) [121] but also causes changes in the zeta-potentials and the $\mathrm{pH}_{\mathrm{IEP}}$ of abrasive particles and films [122], which have an influence on the adsorbed amounts and thickness of dispersants and passivation agents.

(iii) Case III: Since the formation of hydrogen ions and hydroxide ions from water $\left(\mathrm{H}_{2} \mathrm{O} \leftrightarrow \mathrm{H}^{+}+\mathrm{OH}^{-}\right)$is an endothermic process, the water dissociates to $\mathrm{H}^{+}$and $\mathrm{OH}^{-}$ as the temperature increases. The slurry $\mathrm{pH}$ decreases with increasing $\mathrm{H}^{+}$concentration at a higher temperature. Kim et al. reported that the change $(\Delta \mathrm{pH})$ in the $\mathrm{pH}$ of silica and ceria slurries as the temperature increased from 20 to $90{ }^{\circ} \mathrm{C}$ was $\sim 1.0$ and $\sim 1.3$, respectively $[116,119]$. It is well known that a change in $\mathrm{pH}$ of slurry has a significant influence on the particle-wafer interactions [123, 124].

Also, the solubility of oxygen in water decreases with increasing temperature [125]. For example, $10.07 \mathrm{mg} / \mathrm{L}$ of oxygen can be dissolved in the water at $15^{\circ} \mathrm{C}$, while $7.54 \mathrm{mg} / \mathrm{L}$ of oxygen can be dissolved in the water at $30^{\circ} \mathrm{C}$ [125]. The change in the dissolved oxygen concentrations can affect the oxidation and dissolution process of metal films and the surface chemistry of abrasive particles as well as their mutual interactions [3].

(iv) Case IV: The hydrodynamic size $\left(R_{\mathrm{h}}\right)$ of abrasive particles can be determined by the Stokes-Einstein relation; $R_{\mathrm{h}}=$ $k T / 6 \pi \eta D$ where $k, T$, $\eta$, and $D$ represent Boltzmann's constant, temperature, water viscosity, and the diffusion coefficient. Since the equation includes the viscosity of the fluid medium (in our case, water) and the temperature, both parameters strongly influence on the hydrodynamic size of particles. Indeed, the viscosity of the water $\eta$ decreases with increasing temperature (Case V) [126]. Zeta potentials of the abrasive particles increase with temperature. Zeta potentials of particles can be obtained indirectly from the measurement of the electrophoretic mobility according to Henry's equation; $U_{\mathrm{E}}=2 \epsilon \zeta F(\kappa a) / 3 \eta$ where $U_{\mathrm{E}}$ is the electrophoretic mobility, $\epsilon$ is the relative permittivity of water, and $F(\kappa a)$ is Henry's function (dimensionless). For particles in an aqueous medium, $F(\kappa a)$ is 1.5 according to the Smoluchowski approximation. The variation of zeta potential of abrasive particles with temperature is explained by the known temperature dependence of the viscosity $(\eta)$ and the relative permittivity $(\epsilon)$ of water. Evenhuis et al. reported that experimentally determined values of zeta-potential were directly proportional to the temperature [122]. Kim et al. also showed an increase in the zeta-potentials of commercial ceria slurries with increasing slurry temperatures [116]. It may be possible for the number of active particles that participate in material removal during polishing to be changed depending on the particle size and zeta potentials of the abrasive particles.

(v) Case V, VI, and VII: The viscosity of the slurry is a measure of its resistance to deformation by shear forces. High slurry viscosity impedes the transport of the slurry components across the wafer surface. As the temperature of liquid increases, the viscosity of slurry decreases (Case $V$ ), and the reactant molecules move faster (Case VI). Also, according to the Stribeck curve [Fig. 2(b)] [27, 28], a decrease in the slurry viscosity at a higher temperature may allow the direct contact between two solid films with a thin slurry film thickness resulting in an increase in COF during polishing.

\section{In Situ Techniques to Study the Phenomena that Occur at the Slurry/pad-wafer Interface during Polishing}

Research in the field of CMP has significantly increased, as the semiconductor industry grows. Numerous experimental techniques have been employed to understand particle-filmwafer interactions, but they are not adapted for in situ and realtime detection during polishing due to their dimensions and operating requirements. The actual CMP system includes the mutual interactions between chemical and mechanical effects. In situ measurement data collected during polishing are likely to provide more important insights into the underlying mechanisms of various complex reactions that occur at the slurry/ pad-wafer interface. This section offers an overview of advances in the research and developments of in situ measurement studies in CMP systems and then discusses the challenges associated with these measurements. Table 2 presents a summary of in situ measurements applied in CMP systems while highlighting the main applications for each technique.

Slurry film thickness was in situ monitored by analyzing the fluorescence intensities of the two different dye molecules in the slurry using a dual emission UV-enhanced fluorescence technique with a dual-camera imaging system [Fig. 2(a); Table 2] as described in the Hydrodynamic lubrication section 
TABLE 2. Summary of in situ techniques studies in CMP systems.

\begin{tabular}{|c|c|c|}
\hline In situ techniques & Applications & \\
\hline $\begin{array}{l}\text { Dual emission UV-enhanced fluorescence technique with } \\
\text { the dual-camera imaging system }[23,24]\end{array}$ & Slurry film thickness & $\begin{array}{l}\text { Measurement of slurry film thickness between the wafer and the pad } \\
\text { during polish }\end{array}$ \\
\hline Slurry flow visualization setup [127] & Slurry flow & Observation of slurry flow during polishing depending on CMP conditions \\
\hline Rheo-polishing setup [34] & Viscosity & $\begin{array}{l}\text { Measurement of the viscosity and shear thickening of the slurry during } \\
\text { polishing. }\end{array}$ \\
\hline $\begin{array}{l}\text { Surface characterization of wafer-abrasive interactions } \\
\text { using surface-enhanced Raman scattering measurement } \\
\text { [128] }\end{array}$ & Chemical reactions & $\begin{array}{l}\text { Analysis of various chemical interactions between wafer surface and slurry } \\
\text { components }\end{array}$ \\
\hline $\begin{array}{l}\text { ATR-FTIR characterization using mSREs during polishing } \\
\text { [129] }\end{array}$ & $\begin{array}{l}\text { Chemical reactions and } \\
\text { film thickness }\end{array}$ & $\begin{array}{l}\text { Overall chemical reaction characteristics between abrasive particles and } \\
\text { films, change in the surface chemistry of particle, slurries, and film, the } \\
\text { thickness of films. }\end{array}$ \\
\hline $\begin{array}{l}\text { Direct observation of particle single using EW microscopy } \\
\text { [130] }\end{array}$ & $\begin{array}{l}\text { Number of particles, } \\
\text { particle velocity }\end{array}$ & Actual movements of abrasive particles on the wafer surface \\
\hline $\begin{array}{l}\text { Local temperature variation using infrared thermography } \\
\text { [131] }\end{array}$ & Temperature & $\begin{array}{l}\text { In situ observation temperature generated by local material removal } \\
\text { phenomena }\end{array}$ \\
\hline $\begin{array}{l}\text { Electrochemical measurements under controlled } \\
\text { tribological conditions }[60]\end{array}$ & $\begin{array}{l}\text { Electrochemical } \\
\text { reactions }\end{array}$ & $\begin{array}{l}\text { OCP transients, potentiodynamic polarization (the measurement of } \\
\left.\text { corrosion parameters such as } E_{\text {corr }} I_{\text {corr }} I_{\mathrm{g}}, E_{\mathrm{g}}, R_{\mathrm{p}}\right)\end{array}$ \\
\hline
\end{tabular}

$[23,24]$. Mueller et al. [127] used tracer particles to study fluid flow characteristics over pad asperities and recorded a video at 30 frames/s using high definition camera to obtain in situ slurry fluid flow data during polishing (Table 2) and manually analyzed the qualitative information of the slurry flow characteristics from the video. This tool and method enable to study wafer-scale slurry flow visualization depending on CMP conditions, slurry injection locations, and various pad types [127]. The studies that used dyes or tracer particles contain valuable information on slurry film thickness and flow characteristics $[23,24,127]$. However, these particles added to the slurry may not only have a significant influence on the chemical and mechanical reactions during polishing but also become embedded in the pad due to the hydrophobic interactions [127]. Moreover, the materials to be polished are limited to transparent glasses over UV and visible regions.

In situ rheo-polishing setup with a parallel-plate geometry was developed by Crawford et al. [Fig. 8(a); Table 2] [34]. The top was constructed of a disposable plate, while a 2 -in diameter $\mathrm{SiO}_{2}$ blanket wafer was inserted into the fixture housing with $2 \mathrm{~mL}$ of slurry. The gap between the top plate and the wafer surface was $30 \mu \mathrm{m}$, which allowed for shear rates up to 200 , $000 \mathrm{~s}^{-1}$. In some cases, shear-induced aggregation of particles, less than $2 \%$ of the total number of particles, was found at a high shear rate $\left(\geq 10,000 \mathrm{~s}^{-1}\right)$. Shear thickened samples generated about seven times more scratches on the wafer surface than non-thickened slurries. However, as they mentioned, this method not only has no applied normal force but also is incapable of measuring the real changes in slurry viscosity with rolling friction [34].

Takaya et al. have developed a custom-built Raman spectrometer to study various interactions of $\mathrm{Cu}$-abrasive, abrasiveperoxide, BTA-peroxide, and BTA-abrasive-peroxide (Table 2) [128]. The surface-enhanced Raman scattering (SERS) system is an amplification technique that enhances Raman signal from molecules adsorbed on rough metal surfaces. $25 \mathrm{~nm}$ thick $\mathrm{Cu}$ film deposited on $10 \mathrm{~mm}$ glass was used as a substrate for SERS measurements, and it is immersed in the glass cell filled with the slurry. Their SERS analysis results for BTA-abrasive-peroxide suggested that both $\mathrm{Cu}-\mathrm{BTA}$ and $\mathrm{Cu}$ (II)-fullerenol-complex layers are formed on the $\mathrm{Cu}$ surface simultaneously, and these layers may inhibit the further interactions of chelating agents and oxidizer with $\mathrm{Cu}$ film [128]. However, this method is also limited to only chemical reactions without mechanical effects. Since the actual metal polishing system includes the kinetics of competitive reactions between oxidizer, chelating agent, and inhibitor when the passivating layers were removed by mechanical abrasion, mechanical abrasion and slurry flow system, which need to be considered to get more realistic results.

The ATR-FTIR technique for the in situ characterization of CMP using micro-structured single reflection elements (mSREs) was developed by U. Künzelmann and H. Schumacher (Table 2) [129]. They fabricated the microstructure, consists of a periodic array of $\mathrm{V}$-shaped grooves on the backside of a double-side polished 4-in Si wafer with $525 \mu \mathrm{m}$ thickness by wet etch in hot $\mathrm{KOH}$ solution, which is mSRE. This element not only enables to provide single reflection ATR measurement without any collimation but also covers the entire mid-infrared region with a high optical throughput due to the short light path [129]. The coupling structures of the mSRE face downwards, and the pad is pressed from its upper side and rotated against the static mSRE, while the slurry was injected between at the wafer-pad interface. With the depth around $1 \mu \mathrm{m}$, this technique enables to an in situ analysis of the deposition, etching, and surface modification of wafer, pad, and abrasives during polishing [129]. In situ ATR-FTIR spectra of the actual CMP system are very complex and thus 

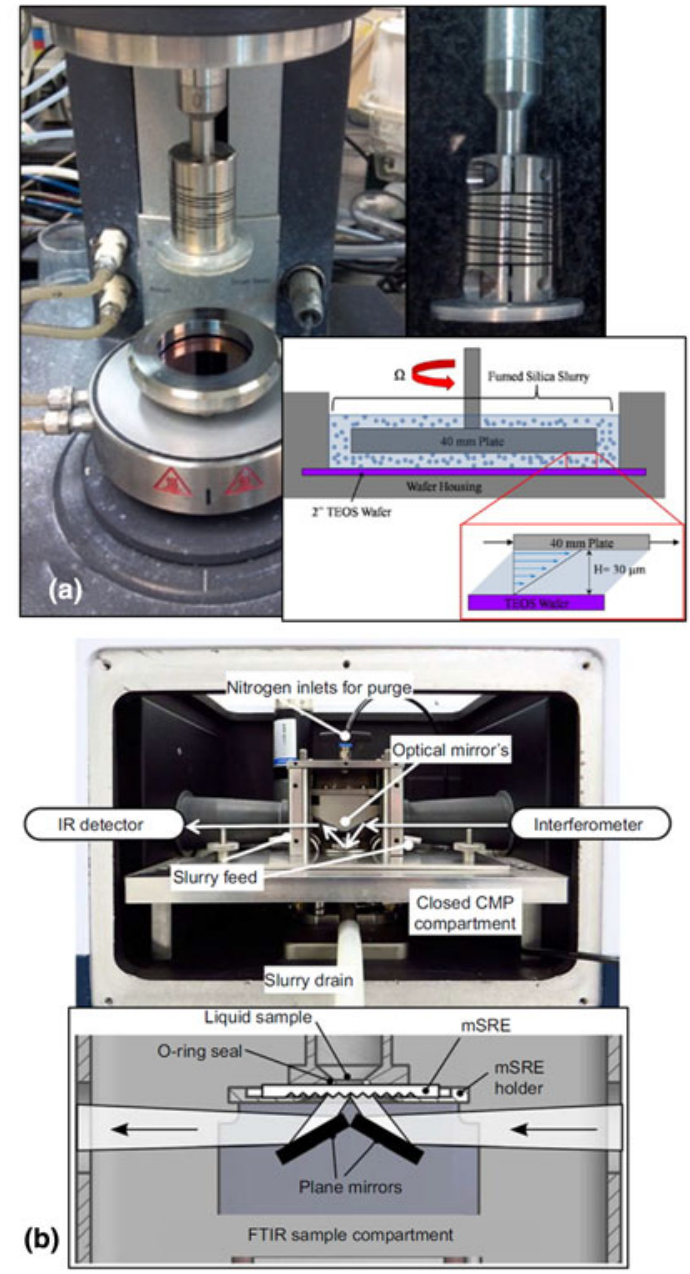

(c)
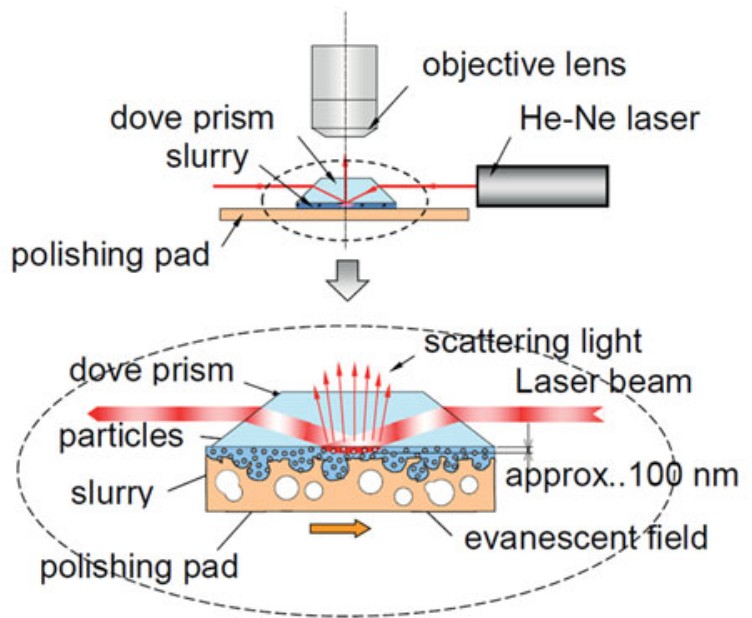

(d)

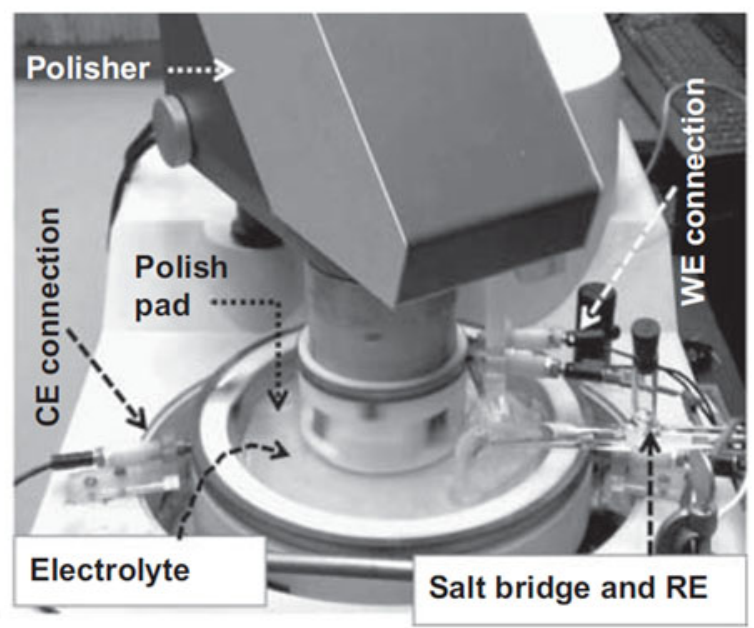

Figure 8: (a) Rheo-polishing setup with plate fixture and $40 \mathrm{~mm}$ upper plate, and schematic of the cross-sectional view of the rheometer plate and tetraethyl orthosilicate (TEOS) wafer covered with CMP slurry. Adapted from Ref. [34]. (b) CMP-ATR accessory with mSRE placed in the sample compartment of a FTIR spectrometer. Adapted from Ref. [129]. (c) Direct observation of particle single using total internal reflection fluorescence. Adapted from Ref. [130]. (d) A three-electrode setup used for electrochemical studies of metal films during polishing. Adapted from Ref. [60].

result in the overlapping absorptions between the main components such as a wafer, pad, and abrasives, which is a major challenge to be addressed by researchers.

Kimura et al. showed that the movement of the particles located at the glass-pad interface could be imaged in situ using evanescent wave (EW) microscopy [Fig. 8(b); Table 2] [130]. EW microscopy, also known as total internal reflection microscopy, coupled with video microscopy was employed to follow the movements of particles near the glass-pad interface. In their experimental setup, an EW was generated when the laser light beam having $30 \mathrm{~mW}$ power at $632 \mathrm{~nm}$ wavelength (He-Ne laser) undergoes total internal reflection at a glassslurry interface and propagated parallel to the surface with an exponentially decaying intensity [130]. The EW illuminates the particles present in the slurry and has a penetration depth $(\sim 100 \mathrm{~nm})$ in an imaged area of $480 \times 640 \mathrm{~mm}^{2}$. The frame rate of the digital high-speed video camera was
60 frames/s (fps). They suggested that the abrasive particles adhered to the $\mathrm{Si}(\mathrm{OH})_{4}$ layer on the $\mathrm{SiO}_{2}$ film were removed by slurry flow, which yields the material removal. They also reported that the actual contact area between the pad and the wafer is less than $2 \%$, and suggested that the significant material removal is caused due to the non-contact lubrication effect [132]. Since Kimura et al. showed a home-built EW imaging system coupled with polishing setup, many research groups have developed different methods to monitor in situ and in real-time the interactions between particle, pad, and wafer during post-CMP cleaning as well as polishing $[133,134]$. Abrasive particles move far away rapidly from the glass surface by the slurry flow and the platen rotation and sometimes disappear from the field of view, preventing long-term particle tracking in the EW field. Moreover, the smaller particles, less than $100 \mathrm{~nm}$ particles used in the actual CMP system, scatter less light making them more challenging to detect using EW 
microscopy [135]. Thus, more light needs to be collected by the objective lens to make the imaging of smaller particles possible.

Local material removal phenomena were in situ observed by infrared thermography (Table 2) [131]. Frictional heating is responsible for increases in the temperature during polishing. Data were obtained from an infrared image sensor with the infrared light collected through a small hole fabricated on the wafer carrier during polishing. An increase in the temperature is related to material removal efficiency. The feasibility of the in situ observation of the polishing efficiency was confirmed by the polishing experiment with a polishing pad. According to Isobe's polishing model [136], they suggested that the in situ detection of instantaneous temperature variations depending on Feret's diameters of the contact area between the pad and the wafer, leading to higher material removal rate [131]. As they mentioned, it is very difficult to directly measure the change in the temperature at the particlewafer-pad interface due to the limitation of the proposed method. Also, the system needs to apply fresh slurry to the pad-wafer interface and remove warm slurry after polishing that passed under the carrier to avoid heat accumulation.

Roy's group reported an electrochemical measurement coupled with a Struers Benchtop Polisher [60]. The top part of the system contains a 1 -in diameter coupon wafer as a working electrode embedded in a Teflon holder attached to the polishing head. And the bottom part is a Teflon container attached to the platen. Electrical connection to the working electrode and the counter electrode was made through carbon brushes pressed against $\mathrm{Cu}$ ring surrounding Teflon holder (Top) and Teflon container (bottom), respectively. The reference electrode equipped with a salt bridge connects to the electrolyte. This system enables to in situ measure OCP transients and potentiodynamic polarization for the metal film under controlled tribological conditions. Roy's group demonstrated that the tribology and mechanical abrasion affects various catalytic reactions of $\mathrm{Cu}$, $\mathrm{Ru}, \mathrm{Ta}$, and Co surfaces during polishing by evaluating CMP systems in the tribo-electrochemical approach [60, 137, 138].

In situ measurements are taken in real-time during polishing and can be interpreted immediately. Moreover, in situ measurements can give more representative information of CMP slurry characteristics, including various chemical and mechanical actions that occur at the slurry/pad-wafer interface during polishing when compared with the ex situ analysis of CMP slurries. However, as stated above, there are still several limitations that need to be addressed. Additional studies and in situ techniques will be helpful to understand the underlying mechanism taking place during the CMP process.

\section{Challenges and Future Directions}

(i) Development of CMP slurries for new materials and integration schemes: As the scaling of the device dimensions runs into physical limitations, 3D integration, 3D NAND technology, and FinFET structure were employed by using new materials and processes [139, 140]. As the architectures have changed from $2 \mathrm{D}$ planar to $3 \mathrm{D}$ vertical, new CMP steps involving new materials and complex 3D structures were proposed. Successful implementation of these schemes required developing novel CMP slurries and other consumables to achieve a range of tunable polish rates for new materials based on their characteristics such as stoichiometry, surface chemistry, hardness, chemical inertness or sensitivity, and thickness. A systematic and effective approach needs to be adopted to achieve the necessary high polishing performance, selective polishing, high planarity, low defects, etc. The research strategies, suggested by the author, for developing next-generation CMP slurries, are summarized in Fig. 9.

(ii) Defect reduction and post-CMP cleaning: Devices at $7 \mathrm{~nm}$ node and beyond present even more stringent requirements for the level of acceptable defectivity during the CMP process. The processes become more defect sensitive and require more expensive metrology techniques with appropriate optical resolution to detect the smaller defects. CMP-induced defects caused by residual particles, foreign materials, scratches, corrosion, etc. should be avoided during polishing. Since this is not always possible, post-CMP cleaning has become a crucial step to eliminate many of these defects $[141,142,143$, 144]. Recently, the author not only showed that the rupture of a strong chemical bonding between abrasive particles and the $\mathrm{SiO}_{2}$ films via a nucleophilic attack could help remove the particles from the surface [141] but also reported a stability constant-based strategy to study reagents that can remove $\mathrm{Cu}-\mathrm{BTA}$ and $\mathrm{Co}-\mathrm{BTA}$ complexes from various surfaces $\left(\mathrm{Cu}, \mathrm{Co}, \mathrm{TaN}\right.$, and $\mathrm{SiO}_{2}$ films) [109, 110]. Fundamental understanding of the formation and characterization of various types of CMP-induced defects will immensely benefit the development of next-generation CMP slurries and post-CMP cleaning solutions.

(iii) Development of environmentally friendly CMP slurries: Azoles such as BTA and TAZ are not only poorly biodegradable under wastewater treatment conditions but also lead to watermarks on the wafer surfaces and contamination of various surfaces under CMP conditions. The effluent wastewater from a semiconductor fabrication plant may contain high concentrations of azoles, etc., and will be challenging for wastewater treatment processes. Hence, the chemical additives that effectively passivate metal surfaces and exhibit sufficiently high biodegradation rates need to be considered keeping their environmental impact in mind. 


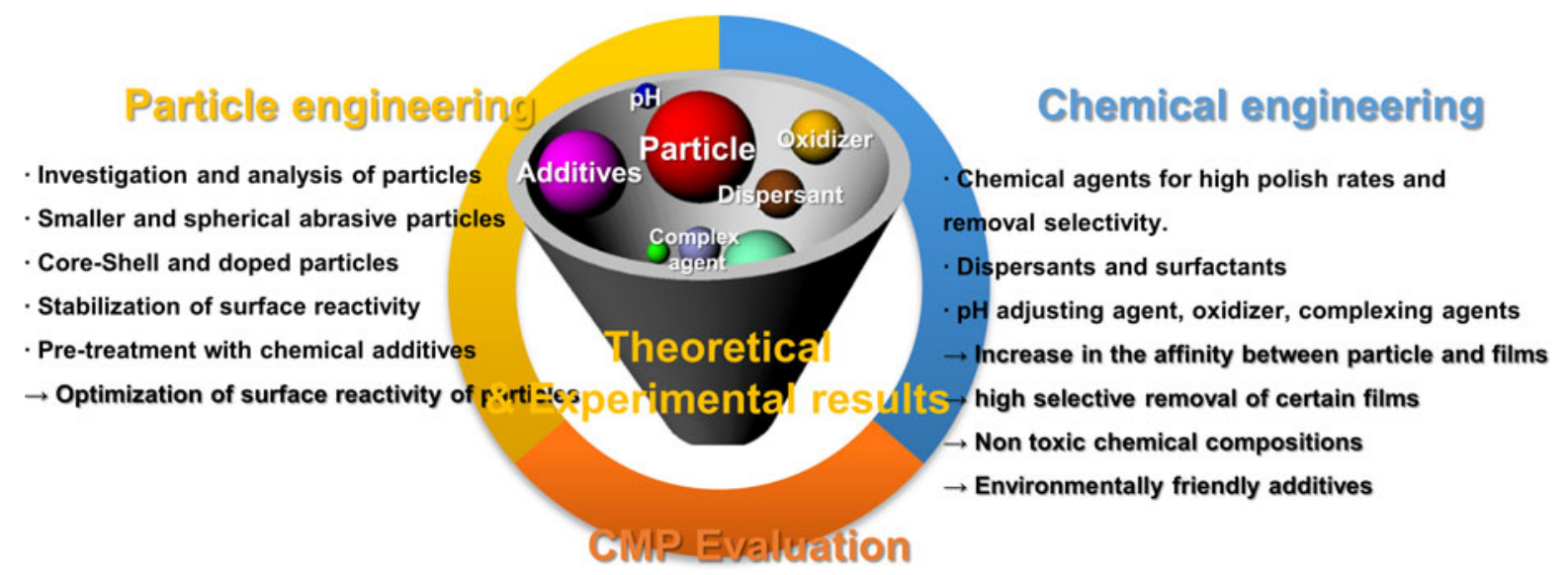

- Removal rate, Removal selectivity, Roughness.

- Surface roughness, low scratches and defects, low dishing

- Optimization of slurry

$\rightarrow$ New slurry compositions for future devices

Figure 9: Schematic diagram of some research strategies for developing next-generation CMP slurries.

(iv) Toxicity issues and safety considerations [145, 146] Toxic gases (e.g., $\mathrm{PH}_{3}, \mathrm{AsH}_{3}$ ) and the As containing waste slurries can be generated during the polishing of III-V materials such as GaAs, InGaAs, InAs, and InP. Hence, the goal of the polishing of III-V materials should be achieving high planarity without generating toxic by-products. In the acidic $\mathrm{pH}, \mathrm{Ru}$ film can be converted to highly volatile $\mathrm{RuO}_{4}$, a toxic gas. The addition of complexing agents such as carboxylic acids can help to reduce gas evolution during polishing. This is an example of tailoring slurry chemistry to minimize the formation of toxic by-products through a careful selection of oxidizer, complexing agent, corrosion inhibitor, and the slurry $\mathrm{pH}$. Tetramethylammonium hydroxide (TMAH), a strong organic base, is also one of the materials showing a high level of concern. TMAH has been widely used as a developer, cleaning agent, $\mathrm{pH}$ adjuster, and etchant in the semiconductor industry, but it can cause fatal damage to nerves and muscles in a short period after exposure by contact [147]. Hence, there has been increasing demand to develop less toxic substitutes for TMAH and improve/control the use of TMAH.

As stated earlier, the challenges and future research directions discussed here were related to CMP slurries and related to chemical and mechanical aspects of the CMP process. There has been significant progress in understanding the fundamental science and technology of CMP for the past several decades. Nevertheless, most of the models, methodologies, and techniques generally use laboratory-scale model systems under the assumptions about process parameters, which may or may not be relevant to explain high-volume-production fabbased CMP processes [148]. A close collaboration between academic and industrial R\&D will help us to move forward with understanding the fundamental principles and technologies of the actual CMP process by minimizing the gaps between labbased CMP models and fab-based CMP processes.

\section{Acknowledgment}

The author gratefully acknowledges Prof. S.V. Babu and Prof. D. Roy for many useful discussions, valuable comments, and suggestions.

\section{References}

1. M. Krishnan, J.W. Nalaskowski, and L.M. Cook: Chemical mechanical planarization: Slurry chemistry, materials, and mechanisms. Chem. Rev. 110, 178 (2009).

2. B. Suryadevara: Advances in Chemical Mechanical Planarization (CMP) (Woodhead Publishing, Cambridge, 2016).

3. R. Srinivasan, P.V. Dandu, and S. Babu: Shallow trench isolation chemical mechanical planarization: A review. ECS J. Solid State Sci. Technol. 4, P5029 (2015).

4. J. Lin, H. Liu, W. Lin, C. Lin, T. Hung, K. Li, J. Lin, J. Wang, C. Liu, and J. Wu: Process development of replacement metal gate Tungsten chemical mechanical polishing on $14 \mathrm{~nm}$ technology node and beyond. In 2015 IEEE International Interconnect Technology Conference and 2015 IEEE Materials for Advanced Metallization Conference (IITC/MAM) (IEEE, San Jose, 2015), p. 115. 
5. V. Kamineni, M. Raymond, S. Siddiqui, F. Mont, S. Tsai, C. Niu, A. Labonte, C. Labelle, S. Fan, and B. Peethala: Tungsten and cobalt metallization: A material study for MOL local interconnects. In Interconnect Technology Conference/ Advanced Metallization Conference (IITC/AMC), 2016 IEEE International (IEEE, San Jose, 2016), p. 105.

6. M. Krishnan and M. Lofaro: Copper chemical mechanical planarization ( $\mathrm{Cu} \mathrm{CMP}$ ) challenges in $22 \mathrm{~nm}$ back-end-of-line (BEOL) and beyond .In Advances in Chemical Mechanical Planarization (CMP), S.V. Babu, ed. (Woodhead Publishing, Cambridge, 2016), pp. 27.

7. J. Seo and U. Paik: Preparation and characterization of slurry for chemical mechanical planarization (CMP) .In Advances in Chemical Mechanical Planarization (CMP), S.V. Babu, ed. (Woodhead Publishing, Cambridge, 2016), pp. 273.

8. H.J. Kim: Abrasive for Chemical Mechanical Polishing .In AbrasiveTechnology: Characteristics and Applications,

A Rudawska, ed. (BoD-Books on Demand, 2018), pp. 183.

9. G. Basim, J. Adler, U. Mahajan, R. Singh, and B. Moudgil: Effect of particle size of chemical mechanical polishing slurries for enhanced polishing with minimal defects. J. Electrochem. Soc. 147, 3523 (2000).

10. J. Luo and D.A. Dornfeld: Effects of abrasive size distribution in chemical mechanical planarization: Modeling and verification. IEEE Trans. Semicond. Manuf. 16, 469 (2003).

11. J. Luo and D.A. Dornfeld: Material removal mechanism in chemical mechanical polishing: Theory and modeling. IEEE Trans. Semicond. Manuf. 14, 112 (2001).

12. H. Lee, H. Jeong, and D. Dornfeld: Semi-empirical material removal rate distribution model for $\mathrm{SiO}_{2}$ chemical mechanical polishing (CMP) processes. Precis. Eng. 37, 483 (2013).

13. C. Lee, $\mathbf{H}$. Lee, $M$. Jeong, and $\mathbf{H}$. Jeong: A study on the correlation between pad property and material removal rate in CMP. Int. J. Precis. Eng. Manuf. 12, 917 (2011).

14. K. Park and H. Jeong: Investigation of pad surface topography distribution for material removal uniformity in CMP process. J. Electrochem. Soc. 155, H595 (2008).

15. L. Borucki: Confocal microscopy pad sample holder that measures displacement and method of using the same. U.S. Patent No. 7,869,027, 2011.

16. Y. Mu, R. Han, Y. Sampurno, Y. Zhuang, and A. Philipossian: Effect of pad surface micro-texture on removal rate during tungsten chemical mechanical planarization. ECS J. Solid State Sci. Technol. 5, P345 (2016).

17. J.C. Mariscal, J. McAllister, Y. Sampurno, J.S. Suarez, L. Borucki, and A. Philipossian: Insights into tungsten chemical mechanical planarization: Part II. Effect of pad surface microtexture on frictional, thermal and kinetic aspects of the process. ECS J. Solid State Sci. Technol. 8, P3175 (2019).
18. H. Lee: Semi-empirical material removal model with modified real contact area for CMP. Int. J. Precis. Eng. Manuf. 20, 1325 (2019).

19. F. Preston: The theory and design of plate glass polishing machines. J. Glass Technol. 11, 214 (1927).

20. W. Fan and D. Boning: Multiscale modeling of chemical mechanical planarization (CMP) .In Advances in Chemical Mechanical Planarization (CMP), S.V. Babu, ed. (Woodhead Publishing, Cambridge, 2016), pp. 137.

21. W.T. Tseng and Y.L. Wang: Re-examination of pressure and speed dependences of removal rate during chemical-mechanical polishing processes. J. Electrochem. Soc. 144, L15 (1997).

22. S. Sundararajan, D.G. Thakurta, D.W. Schwendeman, S.P. Murarka, and W.N. Gill: Two-dimensional wafer-scale chemical mechanical planarization models based on lubrication theory and mass transport. J. Electrochem. Soc. 146, 761 (1999).

23. J. Lu, C. Rogers, V. Manno, A. Philipossian, S. Anjur, and M. Moinpour: Measurements of slurry film thickness and wafer drag during CMP. J. Electrochem. Soc. 151, G241 (2004).

24. C. Gray, D. Apone, C. Rogers, V.P. Manno, C. Barns, M. Moinpour, S. Anjur, and A. Philipossian: Viewing asperity behavior under the wafer during CMP. Electrochem. Solid-State Lett. 8, G109 (2005).

25. D. Rosales-Yeomans, H. Lee, T. Suzuki, and A. Philipossian: Effect of concentric slanted pad groove patterns on slurry flow during chemical mechanical planarization. Thin Solid Films 520, 2224 (2012).

26. Z. Li, H. Lee, L. Borucki, C. Rogers, R. Kikuma, N. Rikita, K. Nagasawa, and A. Philipossian: Effects of disk design and kinematics of conditioners on process hydrodynamics during copper CMP. J. Electrochem. Soc. 153, G399 (2006).

27. R. Stribeck: Kugellager für beliebige Belastungen (Buchdruckerei AW Schade, Berlin, 1901).

28. B. Jacobson: The Stribeck memorial lecture. Tribol. Int. 36, 781 (2003).

29. M.D. Hersey: The laws of lubrication of horizontal journal bearings. J. Wash. Acad. Sci. 4, 542 (1914).

30. B. Mullany and G. Byrne: The effect of slurry viscosity on chemical-mechanical polishing of silicon wafers. J. Mater. Process. Technol. 132, 28 (2003).

31. R. Han, Y. Sampurno, S. Theng, F. Sudargho, Y. Zhuang, and A. Philipossian: Application of the Stribeck+ curve in silicon dioxide chemical mechanical planarization. ECS J. Solid State Sci. Technol. 6, P161 (2017).

32. M. Bahr, Y. Sampurno, R. Han, and A. Philipossian: Improvements in Stribeck curves for copper and tungsten chemical mechanical planarization on soft pads. ECS J. Solid State Sci. Technol. 6, P290 (2017). 
33. W. Lortz, F. Menzel, R. Brandes, F. Klaessig, T. Knothe, and T. Shibasaki: News from the M in CMP-Viscosity of CMP slurries, a constant? MRS Online Proc. Libr. 767, F1.7.1-F1.7.10 (2003).

34. N.C. Crawford, S.K.R. Williams, D. Boldridge, and

M.W. Liberatore: Shear thickening and defect formation of fumed silica CMP slurries. Colloids Surf., A 436, 87 (2013).

35. S.R. Raghavan and S.A. Khan: Shear-induced microstructural changes in flocculated suspensions of fumed silica. J. Rheol. 39, 1311 (1995).

36. A.J. Khanna, S. Gupta, P. Kumar, F.-C. Chang, and R.K. Singh: Quantification of shear induced agglomeration in chemical mechanical polishing slurries under different chemical environments. Microelectron. Eng. 210, 1 (2019).

37. A.J. Khanna, S. Gupta, P. Kumar, F.-C. Chang, and R.K. Singh: Study of agglomeration behavior of chemical mechanical polishing slurry under controlled shear environments. ECS J. Solid State Sci. Technol. 7, P238 (2018).

38. A. Sorooshian, R. Ashwani, H. Choi, M. Moinpour, A. Oehler, and A. Tregub: Effect of particle interaction on agglomeration of silica-based CMP slurries. MRS Online Proc. Libr. 816, K4.9.1K4.9.7 (2004).

39. N.J. Brown, P.C. Baker, and R.T. Maney: Optical polishing of metals. In 25th Annual Technical Symposium (International Society for Optics and Photonics, San Diego, 1982), p. 42.

40. L.M. Cook: Chemical processes in glass polishing. J. Non-Cryst. Solids 120, 152 (1990).

41. E. Laarz, B.V. Zhmud, and L. Bergström: Dissolution and deagglomeration of silicon nitride in aqueous medium. J. Am. Ceram. Soc. 83, 2394 (2000).

42. R.K. Iler: The Chemistry of Silica (Wiley, New York, 1979).

43. T. Hoshino, Y. Kurata, Y. Terasaki, and K. Susa: Mechanism of polishing of $\mathrm{SiO}_{2}$ films by $\mathrm{CeO}_{2}$ particles. J. Non-Cryst. Solids 283, 129 (2001).

44. P.V. Dandu, B. Peethala, and S. Babu: Role of different additives on silicon dioxide film removal rate during chemical mechanical polishing using ceria-based dispersions.J. Electrochem. Soc. 157, H869 (2010).

45. P.V. Dandu, B. Peethala, H. Amanapu, and S. Babu: Silicon nitride film removal during chemical mechanical polishing using ceria-based dispersions. J. Electrochem. Soc. 158, H763 (2011).

46. P.V. Dandu, V. Devarapalli, and S. Babu: Reverse selectivityHigh silicon nitride and low silicon dioxide removal rates using ceria abrasive-based dispersions. J. Colloid Interface Sci. 347, 267 (2010).

47. J. Cheng, S. Huang, Y. Li, T. Wang, L. Xie, and X. Lu: RE (La, $\mathrm{Nd}$ and $\mathrm{Yb}$ ) doped $\mathrm{CeO}_{2}$ abrasive particles for chemical mechanical polishing of dielectric materials: Experimental and computational analysis. Appl. Surf. Sci. 506, 144668 (2020).
48. B. Praveen, B.-J. Cho, J.-G. Park, and S. Ramanathan: Effect of lanthanum doping in ceria abrasives on chemical mechanical polishing selectivity for shallow trench isolation. Mater. Sci. Semicond. Process. 33, 161 (2015).

49. K. Kim, J. Seo, M. Lee, J. Moon, K. Lee, D.K. Yi, and U. Paik: Ce3+-enriched core-shell ceria nanoparticles for silicate adsorption. J. Mater. Res. 32, 2829 (2017).

50. Y. Hu, R. Gutmann, and T. Chow: Silicon nitride chemical mechanical polishing mechanisms. J. Electrochem. Soc. 145, 3919 (1998).

51. S. Raider, R. Flitsch, J. Aboaf, and W. Pliskin: Surface oxidation of silicon nitride films. J. Electrochem. Soc. 123, 560 (1976).

52. R. Iler: The Chemistry of Silica (Wiley-Interscience, New York, 1979).

53. W.G. America and S. Babu: Slurry additive effects on the suppression of silicon nitride removal during CMP. Electrochem. Solid-State Lett. 7, G327 (2004).

54. N.K. Penta, B. Peethala, H. Amanapu, A. Melman, and S. Babu: Role of hydrogen bonding on the adsorption of several amino acids on $\mathrm{SiO}_{2}$ and $\mathrm{Si}_{3} \mathrm{~N}_{4}$ and selective polishing of these materials using ceria dispersions. Colloids Surf., A 429, 67 (2013).

55. S.R. Alety, K.V. Sagi, and S. Babu: Role of $\mathrm{Ce}^{3+}$ ions in achieving high silicon nitride polish rates. ECS J. Solid State Sci. Technol. 6, P898 (2017).

56. F. Kaufman, D. Thompson, R. Broadie, M. Jaso, W. Guthrie, D. Pearson, and M. Small: Chemical-mechanical polishing for fabricating patterned $\mathrm{W}$ metal features as chip interconnects. J. Electrochem. Soc. 138, 3460 (1991).

57. E. Paul: Application of a CMP model to tungsten CMP. J. Electrochem. Soc. 148, G359 (2001).

58. E. Paul, F. Kaufman, V. Brusic, J. Zhang, F. Sun, and R. Vacassy: A model of copper CMP. J. Electrochem. Soc. 152, G322 (2005).

59. E. Paul and R. Vacassy: A model of CMP: III. Inhibitors. J. Electrochem. Soc. 150, G739 (2003).

60. D. Roy: Electrochemical techniques and their applications for chemical mechanical planarization (CMP) of metal films .In Advances in Chemical Mechanical Planarization (CMP), S.V. Babu, ed. (Woodhead Publishing, Cambridge, 2016), pp. 47.

61. D. Lee, H. Lee, and H. Jeong: Slurry components in metal chemical mechanical planarization (CMP) process: A review. Int. J. Precis. Eng. Manuf. 17, 1751 (2016).

62. J. Seo, J.H. Kim, M. Lee, K. You, J. Moon, D.-H. Lee, and U. Paik: Multi-objective optimization of tungsten CMP slurry for advanced semiconductor manufacturing using a response surface methodology. Mater. Des. 117, 131 (2017).

63. T. Du, D. Tamboli, Y. Luo, and V. Desai: Electrochemical characterization of copper chemical mechanical planarization in $\mathrm{KIO}_{3}$ slurry. Appl. Surf. Sci. 229, 167 (2004) 
64. C. Ranaweera, N. Baradanahalli, R. Popuri, J. Seo, and S. Babu: Ammonium persulfate and potassium oleate containing silica dispersions for chemical mechanical polishing for cobalt interconnect applications.ECS J. Solid State Sci. Technol. 8, P3001 (2019).

65. Y. Hong, U. Patri, S. Ramakrishnan, D. Roy, and S. Babu:

Utility of dodecyl sulfate surfactants as dissolution inhibitors in chemical mechanical planarization of copper. J. Mater. Res. 20, 3413 (2005).

66. S. Hegde, U.B. Patri, and S. Babu: Chemical-mechanical polishing of copper using molybdenum dioxide slurry. J. Mater. Res. 20, 2553 (2005).

67. J.-H. Lim, J.-H. Park, and J.-G. Park: Effect of iron (III) nitrate concentration on tungsten chemical-mechanical-planarization performance. Appl. Surf. Sci. 282, 512 (2013).

68. S. Aksu and F.M. Doyle: The role of glycine in the chemical mechanical planarization of copper. J. Electrochem. Soc. 149, G352 (2002)

69. R. Popuri, K. Sagi, S. Alety, B. Peethala, H. Amanapu, R. Patlolla, and S. Babu: Citric acid as a complexing agent in chemical mechanical polishing slurries for cobalt films for interconnect applications. ECS J. Solid State Sci. Technol. 6, P594 (2017).

70. W.-T. Tseng: Approaches to defect characterization, mitigation, and reduction .In Advances in Chemical Mechanical Planarization (CMP), S.V. Babu, ed. (Woodhead Publishing, Cambridge, 2016), pp. 433.

71. C.M. Sulyma and D. Roy: Electrochemical characterization of surface complexes formed on $\mathrm{Cu}$ and $\mathrm{Ta}$ in succinic acid based solutions used for chemical mechanical planarization. Appl. Surf. Sci. 256, 2583 (2010).

72. E. Verink: Simplified procedure for constructing Pourbaix diagrams .In Uhlig's Corrosion Handbook, R. Winston Revie, ed. (John Wiley \& Sons, Vol. 7, Hoboken, NJ, USA, 2011), pp. 111.

73. M.R. Oliver: Chemical-Mechanical Planarization of Semiconductor Materials (Springer Science \& Business Media, Berlin, 2013).

74. B. Peethala, D. Roy, and S. Babu: Controlling the galvanic corrosion of copper during chemical mechanical planarization of ruthenium barrier films. Electrochem. Solid-State Lett. 14, H306 (2011).

75. A.M. Chockalingam, U.R.K. Lagudu, and S. Babu: Potassium periodate-based solutions for minimizing galvanic corrosion at the $\mathrm{Cu}-\mathrm{Mn}$ interface and for polishing the associated $\mathrm{Cu}$ interconnect structures.ECS J. Solid State Sci. Technol. 2, P160 (2013).

76. L. Jiang, Y. He, X. Niu, Y. Li, and J. Luo: Synergetic effect of benzotriazole and non-ionic surfactant on copper chemical mechanical polishing in $\mathrm{KIO}_{4}$-based slurries. Thin Solid Films 558, 272 (2014).
77. S. Farrokhpay: A review of polymeric dispersant stabilisation of titania pigment. Adv. Colloid Interface Sci. 151, 24 (2009).

78. J. Seo, J.W. Lee, J. Moon, W. Sigmund, and U. Paik: Role of the surface chemistry of ceria surfaces on silicate adsorption. ACS Appl. Mater. Interfaces 6, 7388 (2014).

79. S.-K. Kim, S. Lee, U. Paik, T. Katoh, and J.-G. Park: Influence of the electrokinetic behaviors of abrasive ceria particles and the deposited plasma-enhanced tetraethylorthosilicate and chemically vapor deposited $\mathrm{Si}_{3} \mathrm{~N}_{4}$ films in an aqueous medium on chemical mechanical planarization for shallow trench isolation. J. Mater. Res. 18, 2163 (2003).

80. V.A. Hackley: Colloidal processing of silicon nitride with poly (acrylic acid): I, adsorption and electrostatic interactions.J. Am. Ceram. Soc. 80, 2315 (1997).

81. A. Sehgal, Y. Lalatonne, J.-F. Berret, and M. Morvan: Precipitation-redispersion of cerium oxide nanoparticles with poly(acrylic acid): Toward stable dispersions. Langmuir 21, 9359 (2005).

82. P.C. Hidber, T.J. Graule, and L.J. Gauckler: Citric acid-A dispersant for aqueous alumina suspensions.J. Am. Ceram. Soc. 79, 1857 (1996).

83. H. Ohtsuka, H. Mizutani, I. Satoshi, K. Asai, T. Kiguchi, H. Satone, T. Mori, and J. Tsubaki: Effects of sintering additives on dispersion properties of $\mathrm{Al}_{2} \mathrm{O}_{3}$ slurry containing polyacrylic acid dispersant. J. Eur. Ceram. Soc. 31, 517 (2011).

84. M.-G. Song, J.-h. Lee, Y.-G. Lee, and J.-h. Koo: Stabilization of gamma alumina slurry for chemical-mechanical polishing of copper. J. Colloid Interface Sci. 300, 603 (2006)

85. R. Dylla-Spears, L. Wong, P.E. Miller, M.D. Feit, W. Steele, and T. Suratwala: Charged micelle halo mechanism for agglomeration reduction in metal oxide particle based polishing slurries.Colloids Surf., A 447, 32 (2014).

86. W.-T. Tseng, P.-L. Kuo, C.-L. Liao, R. Lu, and J.-F. Lin: Novel polymeric surfactants for improving chemical mechanical polishing performance of silicon oxide. Electrochem. Solid-State Lett. 4, G42 (2001).

87. Y. Pan, X. Lu, G. Pan, Y. Liu, and J. Luo: Performance of sodium dodecyl sulfate in slurry with glycine and hydrogen peroxide for copper-chemical mechanical polishing.

J. Electrochem. Soc. 157, H1082 (2010).

88. J. Seo, K.S. Yoon, J. Moon, K. Kim, W. Sigmund, and U. Paik: Effects of physico-chemical properties between poly(ethyleneimine) and silica abrasive on copper chemical mechanical planarization. Microelectron. Eng. 113, 50 (2014).

89. G.B. Basim, I.U. Vakarelski, and B.M. Moudgil: Role of interaction forces in controlling the stability and polishing performance of CMP slurries. J. Colloid Interface Sci. 263, 506 (2003).

90. C.-W. Cho, S.-K. Kim, U. Paik, J.-G. Park, and W.M. Sigmund: Atomic force microscopy study of the role of molecular weight of 
poly(acrylic acid) in chemical mechanical planarization for shallow trench isolation. J. Mater. Res. 21, 473 (2006).

91. S. Kim, J.-H. So, D.-J. Lee, and S.-M. Yang: Adsorption behavior of anionic polyelectrolyte for chemical mechanical polishing (CMP). J. Colloid Interface Sci. 319, 48 (2008).

92. Y.-H. Kim, S.-M. Lee, K.-J. Lee, U. Paik, and J.-G. Park: Constraints on removal of $\mathrm{Si}_{3} \mathrm{~N}_{4}$ film with conformationcontrolled poly(acrylic acid) in shallow-trench isolation chemical-mechanical planarization (STI CMP). J. Mater. Res. 23, 49 (2008).

93. Y.-H. Kim, Y.-G. Jung, G.S. Yoon, J. Moon, A. Watanabe,

M. Naito, and U. Paik: Non-prestonian behavior of rectangular shaped ceria slurry in shallow trench isolation chemical mechanical planarization. J. Nanosci. Nanotechnol. 12, 2810 (2012).

94. J.-H. Park, H. Cui, J.-Y. Cho, H.-S. Hwang, W.-J. Hwang, U. Paik, H.-G. Kang, N.-J. Kwak, and J.-G. Park: Multiselectivity chemical mechanical polishing for NAND flash memories beyond 32 nm. J. Electrochem. Soc. 157, H607 (2010).

95. J. Seo, J. Moon, S. Moon, and U. Paik: Interpolymer complexes of poly(acrylic acid) and poly(ethylene glycol) for low dishing in STI CMP. Appl. Surf. Sci. 353, 499 (2015).

96. N.K. Penta, H. Amanapu, and S. Babu: Further investigation of slurry additives for selective polishing of $\mathrm{SiO}_{2}$ films over $\mathrm{Si}_{3} \mathrm{~N}_{4}$ using ceria dispersions. ECS J. Solid State Sci. Technol. 4, P5025 (2015).

97. N.K. Penta, H. Amanapu, B. Peethala, and S. Babu: Use of anionic surfactants for selective polishing of silicon dioxide over silicon nitride films using colloidal silica-based slurries. Appl. Surf. Sci. 283, 986 (2013).

98. J.-D. Lee, Y.-R. Park, B.U. Yoon, Y.-P. Han, S. Hah and J.-T. Moon: Effects of nonionic surfactants on oxide-to-polysilicon selectivity during chemical mechanical polishing. J. Electrochem. Soc. 149, G477 (2002).

99. M. Aliofkhazraei: Corrosion Inhibitors, Principles and Recent Applications (BoD-Books on Demand, Norderstedt, 2018).

100. M.A. Malik, M.A. Hashim, F. Nabi, S.A. Al-Thabaiti, and Z. Khan: Anti-corrosion ability of surfactants: A review. Int. J. Electrochem. Sci. 6, 1927 (2011).

101. W. Zhang, Y. Liu, C. Wang, X. Niu, J. Ji, Y. Du, and L. Han: Role of 1, 2, 4-triazole in $\mathrm{Co} / \mathrm{Cu}$ removal rate selectivity and galvanic corrosion during barrier CMP.ECS J. Solid State Sci. Technol. 6, P786 (2017).

102. Y. Mu, M. Zhong, K.J. Rushing, Y. Li, and D.A. Shipp: Benzotriazole as a passivating agent during chemical mechanical planarization of Ni-P alloy substrates. Appl. Surf. Sci. 315, 190 (2014).

103. S. Yang, B. Zhang, Q. Zhang, R. Wang, X. Yu, C. Wang, and Y. Liu: A study of cobalt galvanic and pitting corrosion with combination of BTA and PMP.ECS J. Solid State Sci. Technol. 8 , P416 (2019).

104. J. Cotton: 2nd International Congress on Metallic Corrosion (NACE, New York, 1963).

105. G. Xue, J. Ding, P. Lu, and J. Dong: SERS, XPS, and electroanalytical studies of the chemisorption of benzotriazole on a freshly etched surface and an oxidized surface of copper. J. Phys. Chem. 95, 7380 (1991).

106. K. You, J. Seo, P.J.H. Kim, and T. Song: Control of tungsten protrusion with surface active agent during tungsten chemical mechanical polishing.ECS J. Solid State Sci. Technol. 6, P822 (2017).

107. R. Popuri, H. Amanapu, C. Ranaweera, N. Baradanahalli, and S. Babu: Potassium oleate as a dissolution and corrosion inhibitor during chemical mechanical planarization of chemical vapor deposited Co films for interconnect applications.ECS J. Solid State Sci. Technol. 6, P845 (2017).

108. B.-J. Cho, S. Shima, S. Hamada, and J.-G. Park: Investigation of cu-BTA complex formation during $\mathrm{Cu}$ chemical mechanical planarization process. Appl. Surf. Sci. 384, 505 (2016).

109. J. Seo, S.H. Vegi, C. Ranaweera, N. Baradanahalli, J.-H. Han, D. Koli, and S. Babu: Formation of cobalt-BTA complexes and their removal from various surfaces relevant to cobalt interconnect applications.ECS J. Solid State Sci. Technol. 8, P3009 (2019).

110. J. Seo, S.H. Vegi, and S. Babu: Post-CMP cleaning solutions for the removal of organic contaminants with reduced galvanic corrosion at copper/cobalt interface for advanced $\mathrm{Cu}$ interconnect applications. ECS J. Solid State Sci. Technol. 8, P379 (2019).

111. H. Hocheng, Y.L. Huang, and L.J. Chen: Kinematic analysis and measurement of temperature rise on a pad in chemical mechanical planarization. J. Electrochem. Soc. 146, 4236 (1999).

112. D. White, J. Melvin, and D. Boning: Characterization and modeling of dynamic thermal behavior in CMP. J. Electrochem. Soc. 150, G271 (2003).

113. S. Oh, and J. Seok: Modeling of chemical-mechanical polishing considering thermal coupling effects. Microelectron. Eng. 85, 2191 (2008).

114. Y.-L. Wang, C. Liu, M.-S. Feng, and W.-T. Tseng: The exothermic reaction and temperature measurement for tungsten CMP technology and its application on endpoint detection. Mater. Chem. Phys. 52, 17 (1998).

115. H. Kim, H. Kim, H. Jeong, E. Lee, and Y. Shin: Friction and thermal phenomena in chemical mechanical polishing. J. Mater. Process. Technol. 130, 334 (2002).

116. N.-H. Kim, Y.-J. Seo, and W.-S. Lee: Temperature effects of pad conditioning process on oxide CMP: Polishing pad, slurry characteristics, and surface reactions. Microelectron. Eng. 83, 362 (2006). 
117. V.R.R. Kakireddy: Effect of temperature on copper chemical mechanical planarization (Ph. D. dissertation). University of South Florida, Tampa, Florida, USA (2007).

118. J. Seo, K. You, J. Moon, J.H. Kim, and U. Paik: Communication -Corrosion behavior of tungsten metal gate in the presence of hydrogen peroxide at acidic medium.ECS J. Solid State Sci. Technol. 6, P169 (2017).

119. N.-H. Kim, P.-J. Ko, Y.-J. Seo and W.-S. Lee: Improvement of TEOS-chemical mechanical polishing performance by control of slurry temperature. Microelectron. Eng. 83(2), 286 (2006).

120. S. Mudhivarthi, P.B. Zantye, A. Kumar, A. Kumar,

M. Beerbom, and R. Schlaf: Effect of temperature on tribological, electrochemical, and surface properties during copper CMP. Electrochem. Solid-State Lett. 8, G241 (2005).

121. M. Wiśniewska: Influences of polyacrylic acid adsorption and temperature on the alumina suspension stability. Powder Technol. 198, 258 (2010).

122. C.J. Evenhuis, R.M. Guijt, M. Macka, P.J. Marriott, and P.R. Haddad: Variation of zeta-potential with temperature in fused-silica capillaries used for capillary electrophoresis. Electrophoresis 27, 672 (2006).

123. J.T. Abiade, W. Choi, and R.K. Singh: Effect of $\mathrm{pH}$ on ceriasilica interactions during chemical mechanical polishing. J. Mater. Res. 20, 1139 (2005).

124. J. Seo, A. Gowda, P. Khajornrungruang, S. Hamada, T. Song, and S. Babu: Trajectories, diffusion, and interactions of single ceria particles on a glass surface observed by evanescent wave microscopy. J. Mater. Res., 35, P1-P321 (2020).

125. H. Montgomery, N. Thom, and A. Cockburn: Determination of dissolved oxygen by the Winkler method and the solubility of oxygen in pure water and sea water. J. Appl. Chem. 14, 280 (1964).

126. L. Korson, W. Drost-Hansen, and F.J. Millero: Viscosity of water at various temperatures. J. Phys. Chem. 73, 34 (1969).

127. N. Mueller, C. Rogers, V.P. Manno, R. White, and

M. Moinpour: In situ investigation of slurry flow fields during CMP. J. Electrochem. Soc. 156, H908 (2009).

128. Y. Takaya, M. Michihata, T. Hayashi, R. Murai, and K. Kano: Surface analysis of the chemical polishing process using a fullerenol slurry by Raman spectroscopy under surface plasmon excitation. CIRP Ann. 62, 571 (2013).

129. U. Künzelmann, and H. Schumacher: Characterization of surface processes during oxide CMP by in situ FTIR spectroscopy .In Advances in Chemical Mechanical Planarization (CMP), S.V. Babu, ed. (Woodhead Publishing, 2016), pp. 359.

130. K. Kimura, K. Suzuki, and P. Khajornrungruang: Study on fine particle behavior in slurry flow between wafer and polishing pad as a material removal process in CMP. In ICPT 2012International Conference on Planarization/CMP Technology (IEEE, Grenoble, 2012), p. 1.
131. N. Suzuki, H. Misono, E. Shamoto, S. Goto, H. Yasuda, and Y. Mochizuki: A novel technique for in-situ observation of local polishing efficiency by utilizing infrared thermography. In ICPT (IEEE, Seoul, 2018).

132. K. Kimura, P. Khajornrungruang, K. Suzuki, and E. Okamoto: Study on wafer and polishing pad surface contact with stationary and dynamic behavior. In ICPT Proceedings (IEEE, Phoenix, 2010), p. 19.

133. Y. Terayama, P. Khajornrungruang, K. Suzuki, K. Kusatsu, S. Hamada, Y. Wada, and H. Hiyama: Real time nanoscale cleaning phenomenon observation during PVA brush scrubbing by evanescent field. ECS Trans. 92, 191 (2019).

134. S. Shima, S. Hamada, Y. Wada, C. Takatoh, and A. Fukunaga: Metrologies of abrasive behaviors for understanding and upgrading CMP process. In 2016 International Symposium on Semiconductor Manufacturing (ISSM) (IEEE, Tokyo, 2016), p. 1.

135. P. Khajornrungruang, S. Korkmaz, P. Angshuman, K. Suzuki,

K. Kimura, and S. Babu: Light scattering model for individual sub-100-nm particle size determination in an evanescent field. Jpn. J. Appl. Phys. 55, 06JG02 (2016).

136. A. Isobe, M. Akaji, and S. Kurokawa: Proposal of new polishing mechanism based on Feret's diameter of contact area between polishing pad and wafer. Jpn. J. Appl. Phys. 52, 126503 (2013).

137. X. Shi, D. Simpson, and D. Roy: Tribo-electrochemical characterization of $\mathrm{Ru}$, Ta and $\mathrm{Cu}$ CMP systems using percarbonate based solutions. ECS J. Solid State Sci. Technol. 4, P5058 (2015).

138. M. Turk, X. Shi, D. Gonyer, and D. Roy: Chemical and mechanical aspects of a Co-Cu planarization scheme based on an alkaline slurry formulation.ECS J. Solid State Sci. Technol. 5, P88 (2015).

139. J. Choe: Comparison of $20 \mathrm{~nm} \& 10 \mathrm{~nm}$-class $2 \mathrm{D}$ planar NAND and 3D V-NAND architecture. In Flash Memory Summit (Techinsights, Santa Clara, 2015).

140. S.S. Vegunta: Next Gen CMP challenges in 3D memory architectures. In Semicon West 2018, NCCAVS Users Group (San Francisco, 2018).

141. J. Seo, A. Gowda, and S. Babu: Almost complete removal of ceria particles down to $10 \mathrm{~nm}$ size from silicon dioxide surfaces.ECS J. Solid State Sci. Technol. 7, P243 (2018).

142. A. Gowda, J. Seo, C.K. Ranaweera and S. Babu: Cleaning solutions for removal of $\sim 30 \mathrm{~nm}$ ceria particles from proline and citric acid containing slurries deposited on silicon dioxide and silicon nitride surfaces.ECS J. Solid State Sci. Technol. 9, 044013 (2020).

143. J. Hong, X. Niu, Y. Liu, Y. He, B. Zhang, J. Wang, L. Han, C. Yan, and J. Zhang: Effect of a novel chelating agent on defect removal during post-CMP cleaning. Appl. Surf. Sci. 378, 239 (2016). 
144. S.R. Alety, U.R. Lagudu, R. Popuri, R. Patlolla, C.V. Surisetty, and S. Babu: Cleaning solutions for ultrathin Co barriers for advanced technology nodes. ECS J. Solid State Sci. Technol. 6, P671 (2017).

145. S.V. Babu: CMP: An overview, the good and not so good. In ICPT 2019 (International Conference on Planarization/CMP Technology, Hsinchu, 2019).

146. P. Ong and L. Teugels: CMP processing of high mobility channel materials: Alternatives to Si .In Advances in Chemical Mechanical
Planarization (CMP), S.V. Babu, ed. (Woodhead Publishing, Cambridge, 2016), pp. 119.

147. C.-C. Lin, C.-C. Yang, J. Ger, J.-F. Deng, and D.-Z. Hung: Tetramethylammonium hydroxide poisoning. Clin. Toxicol. 48, 213 (2010).

148. D. Roy: Perspective-Electrochemical assessment of slurry formulations for chemical mechanical planarization of metals: Trends, benefits and challenges. ECS J. Solid State Sci. Technol. 7, P209 (2018). 